\title{
Linear and nonlinear transport across a finite Kitaev chain: An exact analytical study
}

\author{
Nico Leumer $\odot,{ }^{1}$ Milena Grifoni, ${ }^{1}$ Bhaskaran Muralidharan $\odot,{ }^{2}$ and Magdalena Marganska $\odot{ }^{1}$ \\ ${ }^{1}$ Institute for Theoretical Physics, University of Regensburg, 93053 Regensburg, Germany \\ ${ }^{2}$ Department of Electrical Engineering, Indian Institute of Technology Bombay, Powai, Mumbai-400076, India
}

(Received 23 December 2020; revised 2 April 2021; accepted 6 April 2021; published 29 April 2021)

\begin{abstract}
We present exact analytical results for the differential conductance of a finite Kitaev chain in an N-S-N configuration, where the topological superconductor is contacted on both sides with normal leads. Our results are obtained with the Keldysh nonequilibrium Green's function technique, using the full spectrum of the Kitaev chain without resorting to minimal models. A closed formula for the linear conductance is given, and the analytical procedure to obtain the differential conductance for the transport mediated by higher excitations is described. The linear conductance attains the maximum value of $e^{2} / h$ only for the exact zero-energy states. Also, the differential conductance exhibits a complex pattern created by numerous crossings and anticrossings in the excitation spectrum. We reveal the crossings to be protected by inversion symmetry, while the anticrossings result from a pairing-induced hybridization of particlelike and holelike solutions with the same inversion character. Our comprehensive treatment of the Kitaev chain allows us also to identify the contributions of both local and nonlocal transmission processes to transport at arbitrary bias voltage. Local Andreev reflection processes dominate the transport within the bulk gap and diminish for higher excited states but reemerge when the bias voltage probes the avoided crossings. The nonlocal direct transmission is enhanced above the bulk gap but contributes also to the transport mediated by the topological states.
\end{abstract}

DOI: 10.1103/PhysRevB.103.165432

\section{INTRODUCTION}

The search for Majorana zero modes (MZMs) in topological superconductor systems is currently an intensely pursued quest in condensed-matter physics [1-4], with the primary aim to realize a robust framework for topological quantum computing [5-7]. Currently the most advanced experimental platforms for Majorana devices are based on proximitized semiconducting nanowires [8-10], although they have not yet been unambiguously proven to host Majorana states. Transport properties of Majorana nanowire devices have been intensively studied, with the main purpose of devising a detection scheme for the Majorana states by determining their transport fingerprints. The most fundamental one, that of observing a quantized zero bias peak in conductance $[4,11,12]$, can be mimicked by trivial Andreev bound states [10,13-16] or level repulsion in multiband systems $[17,18]$, thus several detection schemes also exploiting Majorana nonlocality have been proposed [19-21]. From the point of view of the applications, one of the schemes for the readout of Majorana qubits is based on transport interferometry [22,23], providing further motivation to explore the transport properties of Majorana devices.

Most of the works in this domain are, out of necessity, either numerical or based upon a minimal model, concentrating on charge transport through the in-gap states [10,1315,24-27]. Our aim is to find an analytical expression for the current flowing through a topological superconductor, taking into account its full excitation spectrum. The knowledge of such analytical solutions for at least one topological superconductor is instrumental in testing the reliability of the numerical results. As our model system, we take a prototypical topological superconductor, the Kitaev chain [28]. Although the low-energy spectrum of a Kitaev chain with two Majorana states has served as the basis for minimal models of nanowire transport, we are aware of only a few analytical studies which focused on the transport characteristics of the Kitaev chain itself, achieving its description in analytical terms for several parameter ranges [29,30]. Doornenbal et al. [29] treated the chain as a fragment of an N-S-N system, but with the bias drop occurring at one contact only, which yields the wellknown value $2 e^{2} / h$ for the conductance through an MZM. Without a self-consistent calculation, it also leads, however, to nonconservation of current. Another recent work [30] studied the low-energy transport properties of a Kitaev chain with long-range superconducting pairing, using a Green's function technique combined with the scattering matrix approach. The transport calculation is analytical, although it needs the eigenvectors as input, which are obtained from a numerical diagonalization of the Hamiltonian.

In this paper, we use the Keldysh nonequilibrium Green's functions technique (NEGF) and the notion of Tetranacci polynomials to derive analytical expressions for both the current and conductance of a Kitaev chain in an N-S-N configuration, in the linear as well as nonlinear transport regime, for arbitrary hopping $t$, superconducting pairing $\Delta$, and chemical potential $\mu$. Thus, we can access not only the known transport properties of the topological states but also of the higher excited states.

While we derive the differential conductance for arbitrary bias drop at the contacts, we show only the results for 
symmetric bias-the configuration in which the current is conserved. In consequence, crossed Andreev reflection processes do not contribute to transport. For the chosen symmetric setup, the transport occurs via two mechanisms, local Andreev reflection and nonlocal direct transmission. Both contributions feature conductance peaks resonant with the excitation energies but with different weights. The transport within the bulk gap is dominated by the local, Andreev processes, while the main contribution to transport above the bulk gap comes from the nonlocal, direct transmission. The excitation spectrum above the bulk gap contains several series of crossings and avoided crossings, ubiquitous in the spectra of Majorana nanowires [13,15,17,27,31-33]. Our analysis sheds light on the nature of these states' features. We find that the crossings are protected by the inversion symmetry of the normal chain - the degenerate eigenstates have particle (hole) sectors of opposite inversion character. On the other hand, the particle (hole) sectors of anticrossing states match under inversion and the superconducting pairing allows the particlelike and holelike solutions of the linear chain to hybridize. Inside the anticrossings, the Andreev reflection processes are revived, reminiscent of their importance in the subgap transport. Similarly, even though the direct transmission plays the prominent role in high bias conductance, it is also responsible for some of the current flowing through the two topological states at low bias. We obtain the maximum value of $e^{2} / h$ for the zero-bias Majorana conductance peak, as expected from an N-S-N setup with symmetric bias drop [25,34,35]. Remarkably, our results show that for a finite chain, the value $e^{2} / h$ for the linear conductance is not obtained in the whole topological phase but only near the Kitaev points $(\mu=0,|t|=|\Delta|)$. Elsewhere, the conductance can be close to its maximum value or even significantly lower.

This paper is organized as follows. First, we analyze the spectrum of an isolated Kitaev chain in Sec. II, including the higher excitations. In Sec. III, we discuss our N-S-N transport setup, providing a general current formula for our system. The analytical expression for the linear conductance is derived in Sec. IV. In Sec. V, we present the formula for the differential conductance at finite bias in terms of appropriate Green's functions. The detailed derivations of the expressions for the current, conductance, and the Green's functions are given in the Appendices.

\section{THE ISOLATED KITAEV CHAIN}

The central element of our N-S-N system is the finite Kitaev chain, which is a tight-binding chain of $N$ lattice sites, with one spinless fermionic orbital at each site and nearestneighbor $p$-wave superconducting pairing. The $p$-wave nature of the superconductivity couples particles of equal spin, allowing a spinless treatment. The pairing is treated in the usual mean-field approach, yielding the Kitaev grandcanonical Hamiltonian $[4,28]$

$$
\begin{aligned}
\hat{H}_{\mathrm{KC}}:= & \hat{H}_{0}-\mu \hat{N}_{\mathrm{KC}}=-t \sum_{j=1}^{N-1}\left(d_{j+1}^{\dagger} d_{j}+d_{j}^{\dagger} d_{j+1}\right) \\
& -\Delta \sum_{j=1}^{N-1}\left(d_{j}^{\dagger} d_{j+1}^{\dagger}+d_{j+1} d_{j}\right)-\mu \sum_{j=1}^{N} d_{j}^{\dagger} d_{j},
\end{aligned}
$$

in terms of the fermionic creation (annihilation) operators $d_{j}^{\dagger}$ $\left(d_{j}\right)$. The quantities introduced in Eq. (1) are the real space position index $j=1, \ldots, N$, the hopping amplitude $t \in \mathbb{R}$, and the superconducting pairing constant $\Delta \in \mathbb{R}$. The action of the gate voltage applied later to the wire is to change the chemical potential as $\mu \rightarrow \mu+\mu_{g}$, with $\mu_{g}=\eta_{g} e V_{g}$, and $\eta_{g}$ the lever arm of the junction.

The spectrum and topological properties of both finite and infinite Kitaev models were discussed in detail in the recent past $[3,4,28,36-39]$ and we shall give here only a brief overview of the low-energy spectrum, giving more emphasis to the hitherto largely unexplored quasiparticle states at higher energy.

In the thermodynamic limit $(N \rightarrow \infty)$, the energy of the excitations obeys the bulk dispersion relation

$$
E_{ \pm}(k)= \pm \sqrt{[\mu+2 t \cos (k d)]^{2}+4 \Delta^{2} \sin ^{2}(k d)},
$$

where $d$ is the lattice constant. The topological features of the Kitaev chain can be found after a calculation of the winding number or the Pfaffian topological invariant [40,41]. The boundaries between trivial and nontrivial phases in the topological phase diagram $[3,4,28]$ are determined by the gap closing of the bulk dispersion relation, which happens at $k=0$ or $k=\pi / d$ for $\Delta \neq 0$ and $\mu= \pm 2 t$. As one finds, the nontrivial phase exists only for $|\mu / \Delta|<2|t / \Delta|$.

In a finite chain, the bulk-edge correspondence $[42,43] \mathrm{im}$ plies the existence of evanescent state solutions at the system's boundary in the topologically nontrivial phase. These states have a complex wave vector $\kappa$, and their wave functions decay away from the edges with a decay length $\xi$, which for $\mu=0$ is given by

$$
\xi=\frac{2 d}{|\ln | \frac{t-\Delta}{t+\Delta}||} .
$$

The energy of these topological excitations lies inside the bulk gap introduced with Eq. (2) and is in general nonzero, with the upper bound proportional to $\exp (-N d / \xi)$; i.e., for $\xi \ll N d$ the edge state energy is exponentially small. ${ }^{1}$ The energy of the decaying states becomes exactly zero for specific parameter settings [36-39,44], namely,

$$
\mu_{n}=2 \sqrt{t^{2}-\Delta^{2}} \cos \left(\frac{n \pi}{N+1}\right),
$$

with $n=1, \ldots, N$ and for $t^{2} \geqslant \Delta^{2}$. Zero-energy solutions for $t^{2}<\Delta^{2}$ are found only for $n=(N+1) / 2$, which is only possible for odd $N$. The zero energy solutions form lines in the $(t / \Delta, \mu / \Delta)$ plane (we shall call them Majorana lines) departing from the points $|t|=|\Delta|, \mu=0$, as depicted in Fig. 1.

The exact zero-energy solutions of the isolated Kitaev chain represent fermion parity switches [36,45-47] and, for given $t, \Delta$ occur for discrete values of $\mu$. Although the finite size effects may raise the energy of the topological states so high that they do not contribute to the zero-bias conductance (see, e.g., the low conductance regions in Fig. 5), close to the

\footnotetext{
${ }^{1}$ The decay length in Eq. (3) is defined for $\mu=0$, since the effect of $\mu$ on $\xi$ is not significant $[28,39]$.
} 


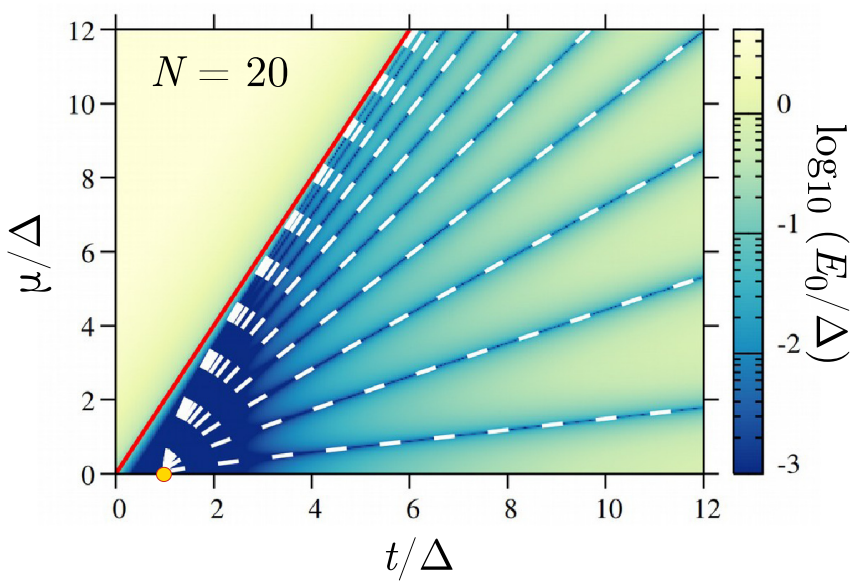

FIG. 1. Lowest positive excitation energy of the isolated Kitaev chain as a function of $t / \Delta$ and $\mu / \Delta$. The color map in the background displays the numerically calculated energy of the lowest eigenstate, the red line depicts the phase boundary $|\mu|=2|t|$ between the topologically trivial $(|\mu|>2|t|)$ and nontrivial $(|\mu|<2|t|)$ bulk phases, while the white dashed lines are the Majorana lines defined by Eq. (4). Along these lines, the ground-state energy $E_{0}$ is exactly zero. All Majorana lines start from the $\mu=0, t= \pm \Delta$ points and are located in the bulk topological region.

Majorana lines the energy of these states is very low, as seen in Fig. 1. Thus, due to the broadening of the energy levels induced by the coupling to the leads, also states with energy smaller than such broadening will effectively act as MZMs. As we shall show, in an N-S-N setup with symmetric bias, they yield a linear conductance very close to $e^{2} / h$, reaching the exact $e^{2} / h$ in the thermodynamic limit $[25,34,35]$. We recall here that in an N-S configuration, the height of the zero bias peak is expected to be $2 e^{2} / h[4,10]$.

\section{A. Higher excitation spectrum}

The low-energy states of the topological superconductors have so far garnered the most attention of the scientific community. Nevertheless, a current flowing through the Kitaev chain at a larger bias will also involve the higher lying excitations. Thus, some questions naturally arise, such as: How will the high-energy spectrum impact the differential conductance? If a chain is in the topological phase, will this affect the features visible at finite bias? To answer these questions, we first analyze the full spectrum of a finite Kitaev chain. The numerically obtained spectrum as a function of $\mu$ is shown in Fig. 2(a) for $t>\Delta$, and in Fig. 2(b) for $\Delta>t$. The eigenstates in the Bogoliubov-de Gennes (BdG) representation are composed of particle $(u)$ and hole $(v)$ components. In most of the spectrum, the eigenstates have either particle $(|u|>|v|)$ or hole $(|v|>|u|)$ character; the states within the bulk gap (cf. Appendix A), but also some higher energy solutions described below, are nearly equal mixtures of both.

The linear chain, which is the foundation of the Kitaev chain in Eq. (1) has inversion symmetry. For the linear chain, the inversion corresponds to a straightforward exchange $d_{j}^{(\dagger)} \rightarrow d_{N+1-j}^{(\dagger)}$ and its matrix representation is an $N \times N$ matrix $I_{0}$ with 1 on the antidiagonal and 0 elsewhere.
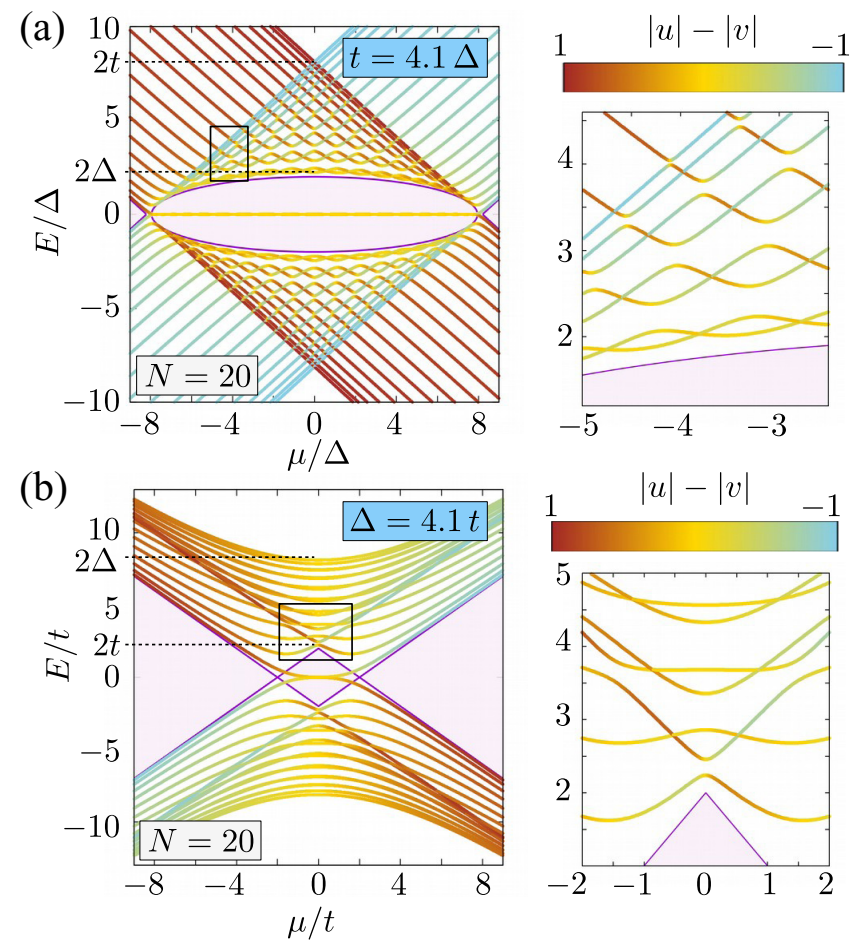

FIG. 2. Full spectrum of a Kitaev chain, for (a) $t=4.1 \Delta$ and (b) $\Delta=4.1 t$. The color scale shows the particle/hole character of the corresponding eigenstate, expressed through $|u|-|v|$, where $|u|$ and $|v|$ are the norms of the particle and hole parts of the eigenstate, respectively. The violet shaded regions show the bulk gap.

If this operation is extended directly to the Kitaev chain, it results in changing the sign of the superconducting pairing because of its $p$-wave nature. The unitary symmetry inverting the order of the sites, under which the Kitaev Hamiltonian is invariant, is instead $d_{j} \rightarrow i d_{N+1-j}, d_{j}^{\dagger} \rightarrow-i d_{N+1-j}^{\dagger}$. Its matrix representation is $I_{\mathrm{KC}}=i \tau_{z} \times I_{0}$, where $\tau_{z}$ is the Pauli matrix $z$ in the Nambu space. Crucially, $I_{\mathrm{KC}}$ applied to a Nambu spinor adds a global phase $i$ (which can later be gauged away) and changes the sign of the hole part of the spinor. In consequence, the particle and hole sectors in each eigenstate of the Kitaev chain must have opposite character under the simple inversion symmetry $I_{0}$ (cf. Fig. 3, for a detailed discussion in a slightly different approach see Appendix D).

For $t>\Delta$, we see a series of anticrossings between the higher excitations, which occur throughout the spectrum. The particlelike and holelike solutions of the normal chain in the Nambu space at the anticrossings have the same character under inversion, thus they can hybridize under the influence of the superconducting pairing. In consequence, the particle and hole sectors of the hybridized quasiparticle eigenstates have nearly equal weight. The crossings, on the other hand, are protected by the different inversion symmetries of the involved eigenstates, which have predominantly particleor holelike character. For $\Delta>t$, the character of the excitation spectrum is naturally different-higher absolute value of $\mu$ again separates the spectrum into particle- and hole-like sets of states, but at $\mu=0$ the particle-hole mixing occurs within the whole spectrum. Unlike in the $t>\Delta$ case, both the strict and avoided crossings now also occur outside of the 

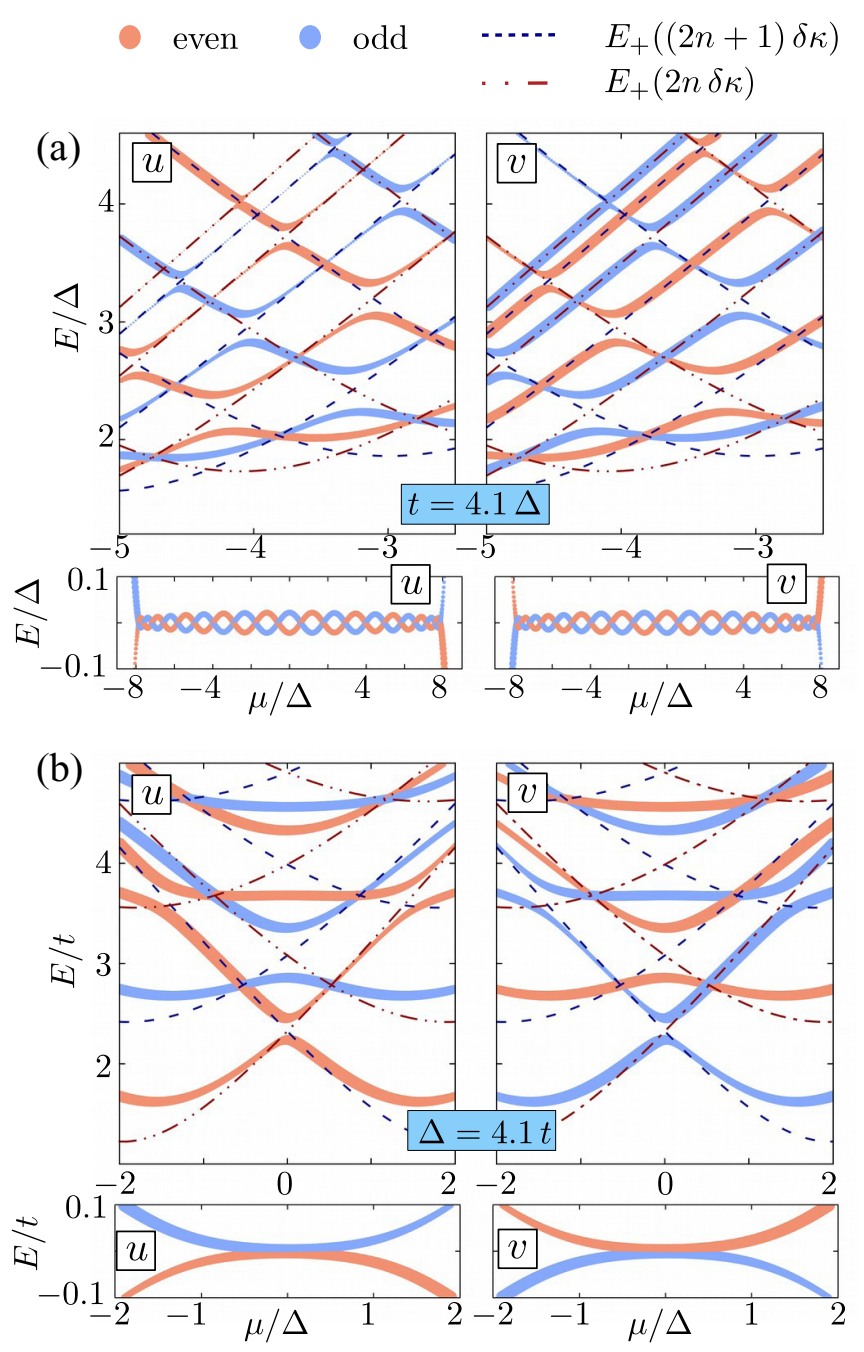

FIG. 3. Inversion symmetry for chosen ranges of the full spectrum, for (a) $t=4.1 \Delta$ and (b) $\Delta=4.1 t$. The color represents $I_{u}:=$ $\left\langle u\left|I_{0}\right| u\right\rangle /|u|^{2}$ for the particle sector and $I_{v}:=\left\langle v\left|I_{0}\right| v\right\rangle /|v|^{2}$ for the hole sector-light red for $u / v$ even under inversion $\left(I_{u / v}=+1\right)$, blue for $u / v$ odd under inversion $\left(I_{u / v}=-1\right)$. Thickness of the lines is proportional to $|u|$ and $|v|$ in the corresponding panel, and the dark dashed and dot-dot-dashed lines follow $E_{+}((2 n+1) \delta \kappa)$ and $E_{+}(2 n \delta \kappa)$ from Eq. (2), respectively, with $\delta \kappa:=\pi /(N+1)$.

topological phase, under the action of the hopping, rather than of the pairing term.

To find the values of $\mu$ at which an eigenvalue $E$ is degenerate, we have to revisit the general quantization rule for the wave vectors of the finite Kitaev chain. As we showed in Ref. [39], the eigenstates of the Kitaev chain require, in general, the knowledge of four wave numbers $\pm \kappa_{1,2}\left(\kappa_{1} \neq \pm \kappa_{2}\right)$, since one has to satisfy two boundary conditions for electron and hole sectors separately. We use $\kappa_{\Sigma}:=\left(\kappa_{1}+\kappa_{2}\right) / 2, \kappa_{\Delta}:=$ $\left(\kappa_{1}-\kappa_{2}\right) / 2$ for shortness. The values of $\kappa_{1,2}$ are related and obey

$$
\cos \left(\kappa_{\Sigma}\right) \cos \left(\kappa_{\Delta}\right)=-\frac{1}{2} \frac{\mu t}{t^{2}-\Delta^{2}},
$$

which can be obtained from Eq. (2) by demanding $E\left(\kappa_{1}\right)=$ $E\left(\kappa_{2}\right)$. Thus, Eq. (5) is in fact a bulk property of the system which also encodes the dependence of $\kappa_{\Sigma}, \kappa_{\Delta}$ on the chemical potential in a finite system. Together with the boundary conditions, it yields the quantization rule of the finite Kitaev chain,

$$
\frac{\sin ^{2}\left[\kappa_{\Sigma}(N+1)\right]}{\sin ^{2}\left[\kappa_{\Delta}(N+1)\right]}=\frac{1+\left(\frac{\Delta}{t}\right)^{2} \cot ^{2}\left(\kappa_{\Delta}\right)}{1+\left(\frac{\Delta}{t}\right)^{2} \cot ^{2}\left(\kappa_{\Sigma}\right)} .
$$

While a detailed derivation of the position of strict and avoided crossings for $E \neq 0$ can be found in Appendix D 3, let us here summarize its results. The boundary conditions, together with the requirement of double degeneracy (higher degeneracies occur only for the special cases of either $t=0$ or $\Delta=0$ or $t^{2}=\Delta^{2}$ ), constrain $\kappa_{\Sigma, \Delta}$ to be selected zeros of $\sin ^{2}\left[\kappa_{\Sigma, \Delta}(N+1)\right]$ :

$$
\begin{gathered}
\kappa_{\Sigma}=\frac{n \pi}{N+1} \quad \text { or } \frac{(N+1-n) \pi}{N+1}, \quad n=2, \ldots, N_{\max }, \\
\kappa_{\Delta}=\frac{m \pi}{N+1}, \quad m=1, \ldots, n-1,
\end{gathered}
$$

with $N_{\max }=N / 2\left(N_{\max }=(N+1) / 2\right)$ for even (odd) $N>$ 3 . These values indeed satisfy the boundary conditions since $\sin ^{2}\left[\kappa_{\Sigma, \Delta}(N+1)\right]\left[1+(\Delta / t)^{2} \cot ^{2}\left(\kappa_{\Sigma, \Delta}\right)\right]=0$, for both $t \gtrless \Delta$. For odd $N$, we find additional $(N-1)$ degeneracies at $\mu=0$, [39] corresponding to $(N-1) / 2$ allowed values for $\kappa_{\Delta}$ if $\kappa_{\Sigma}=\pi / 2$, for both $E \gtrless 0$. The values of $\mu$ where the crossings occur follow from the Eq. (5) for fixed values of $t$ and $\Delta$. While for both $\Delta>t$ and $\Delta<t$ the number of crossings is the same, their positions are not. The energy eigenvalues follow, as usual, from the dispersion relation in Eq. (2).

The conditions for degenerate energy levels are illustrated in Fig. 3. The energies corresponding to $\kappa_{1,2}=n_{1,2} \pi /(N+$ 1) are shown with dashed and dot-dot-dashed lines for odd and even $n_{1,2}$, respectively. The conditions Eqs. (7) and (8) are obeyed at the intersections of the lines with $n_{1,2}$ either both even or both odd, and indeed at these intersections we see strict crossings. On the other hand, the avoided crossings appear for $n_{1,2}$ with different parities. In these cases, $\kappa_{\Sigma, \Delta}$ are not integer, but half-integer multiples of $\pi /(N+1)$, and the quantization rule Eq. (6) implies

$$
1+\left(\frac{\Delta}{t}\right)^{2} \cot ^{2} \kappa_{\Sigma}=1+\left(\frac{\Delta}{t}\right)^{2} \cot ^{2} \kappa_{\Delta},
$$

which can be fulfilled only if $\Delta=0$ because $\kappa_{\Delta} \neq\left.\kappa_{\Sigma}\right|_{\bmod \pi}$. Hence, for $\Delta \neq 0$, these crossings are avoided. Interestingly, the values of $E$ and $\mu$ at their centers can be correctly calculated from Eqs. (2) and (5) by using $\kappa_{\Sigma, \Delta}$ which are half-integer multiples of $\pi /(N+1)$.

One can summarize that the $E \neq 0$ crossings and anticrossings follow the equidistant quantization of a linear chain, where $\Delta=0$ in Eq. (1), but the specific values of $\mu$ and the related energies depend on the nonzero $\Delta$. The presence of crossings and anticrossings is not per se a signature of the topological phase-they can also be found in the nontopological regime for $\Delta>t$, cf. Fig. 2(b). In this case, the crossover into the topological phase introduces only a quantitative change in the higher excitation spectrum-two of the extended states localize, becoming the boundary modes. 


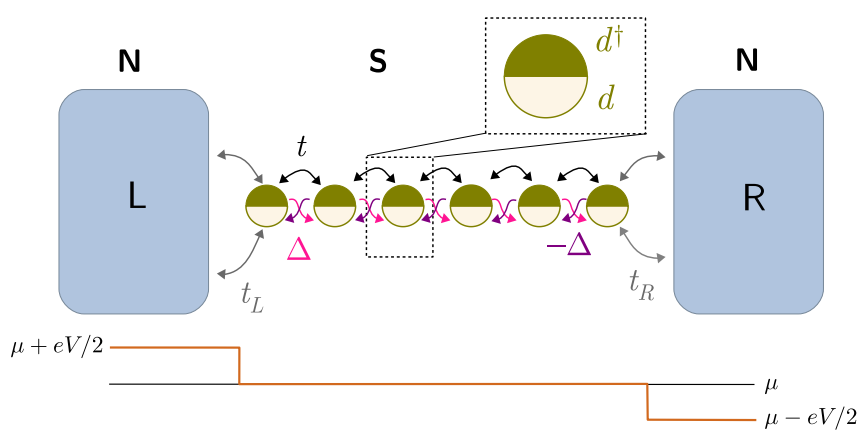

FIG. 4. The Kitaev chain in N-S-N transport configuration, sketched in the particle-hole basis. The parameters $t_{L}, t_{R}$ are the tunneling hoppings between the leads and the Kitaev chain from Eqs. (10). The results shown in this paper are obtained for a symmetrically applied bias. The resulting profile of the chemical potential for the electronic sector is shown below the device.

The energies and wave functions of the remaining extended states readjust to accommodate the presence of the topological states, although for the extended states this change is continuous.

\section{N-S-N TRANSPORT AND THE CURRENT FORMULA}

In this section, we introduce our transport setup, illustrated schematically in Fig. 4, and discuss the current formula. We place the Kitaev chain between two normal conducting leads, described by the grand-canonical Hamiltonians

$$
\hat{H}_{\alpha}-\mu \hat{N}_{\alpha}=\sum_{k} \epsilon_{k \alpha} c_{k \alpha}^{\dagger} c_{k \alpha}, \quad \alpha=L, R,
$$

where $c_{k \alpha}^{\dagger}\left(c_{k \alpha}\right)$ creates (destroys) a spinless fermion in state $k$ and lead $\alpha$. Note that $\hat{H}_{\mathrm{KC}}$ in Eq. (1) and $\hat{H}_{\alpha}$ in Eq. (9) are written with the reference energy being the chemical potential $\mu$ of the Kitaev chain. In the above description, we consider leads in their eigenbasis.

Our N-S-N junction is completed with the tunneling Hamiltonian,

$$
\begin{aligned}
\hat{H}_{\mathrm{T}}= & \sum_{k}\left(t_{L} c_{k L}^{\dagger} d_{1}+t_{L} d_{1}^{\dagger} c_{k L}\right) \\
& +\sum_{k}\left(t_{R} c_{k R}^{\dagger} d_{N}+t_{R} d_{N}^{\dagger} c_{k R}\right)
\end{aligned}
$$

which couples only the first (last) chain site to the contact $L(R)$. We consider the tunneling elements $t_{L, R}$ as $k$ dependent, real quantities. This setup is equivalent to a fully spin polarized system where the fixed spin $\sigma$ is suppressed in the notation.

The current through an N-S-N junction can be calculated with the NEGF approach [48-56]. Since the Kitaev Hamiltonian in Eq. (1) is given in mean field, and thus breaks the conservation of particles, the calculation has to be carried out with care. The physical current measured in experiment is conserved everywhere, thus we calculate it for simplicity inside the left lead. There, the electronic current (for fixed spin) reads

$$
I_{L}(t)=-e\left\langle\dot{N}_{L}\right\rangle
$$

where $e$ is the elementary charge and $N_{L}=\sum_{k} c_{k L}^{\dagger} c_{k L}$. The steady state current (for fixed spin) reads

$$
I_{L}=i \frac{e}{2 h} \int_{\mathbb{R}} d E \operatorname{Tr}\left\{\tau_{z} \otimes \mathbb{1}_{N} \Gamma_{L}\left[G^{<}+F_{L}\left(G^{r}-G^{a}\right)\right]\right\},
$$

with $\Gamma_{\alpha}=-2 \operatorname{Im}\left(\Sigma_{\alpha}^{r}\right)(\alpha=L, R)$, where $\Sigma_{\alpha}^{r}$ is the retarded self-energy of the lead $\alpha$, defined in Appendix E. Since we are working in the $\mathrm{BdG}$ formalism, all quantities under the trace in the above equation are $2 N \times 2 N$ matrices, defined with respect to $\hat{\Psi}=\left(d_{1}, \ldots, d_{N}, d_{1}^{\dagger}, \ldots, d_{N}^{\dagger}\right)^{\mathrm{T}}$, forming electronic and hole subspaces. For example, the matrix $F_{\alpha}$ contains the Fermi functions $f(E)$ for electrons and holes in the following form:

$$
F_{\alpha}=\left[\begin{array}{ll}
\mathbb{1}_{N} f\left(E-e V_{\alpha}\right) & \\
& \mathbb{1}_{N} f\left(E+e V_{\alpha}\right)
\end{array}\right],
$$

where $V_{L}=\eta V, V_{R}=(\eta-1) V$ account for scenarios with different applied bias $V$. Further, the lesser Green's function $G^{<}$is

$$
G^{<}=i G^{r}\left(\sum_{\alpha=L, R} F_{\alpha} \Gamma_{\alpha}\right) G^{a}
$$

with details of the derivation discussed in Appendix E. In equilibrium $(V=0)$, we have $G_{\text {eq }}^{<}=-F(E)\left(G^{r}-G^{a}\right)$ and the current vanishes.

The special choice of the tunneling Hamiltonian in Eqs. (10) defines the self-energies $\Sigma_{\alpha}^{r}$ as sparse matrices, see Eqs. (F4) and (F5). This, together with the trace and the particle-hole symmetry, yields a current formula where only two entries of the retarded Green's function, namely, $G_{1, N}^{r}$ and $G_{1, N+1}^{r}$, are required. One finds

$$
\begin{aligned}
I_{L}= & \frac{e}{h} \int_{\mathbb{R}} d E\left\{\Gamma_{L}^{-} \Gamma_{R}^{-}\left|G_{1, N}^{r}\right|^{2}\left[f\left(E-e V_{L}\right)-f\left(E-e V_{R}\right)\right]\right. \\
& \left.+\Gamma_{L}^{-} \Gamma_{L}^{+}\left|G_{1, N+1}^{r}\right|^{2}\left[f\left(E-e V_{L}\right)-f\left(E+e V_{L}\right)\right]\right\},
\end{aligned}
$$

now setting $V_{L}=-V_{R}=V / 2$. We choose this scenario to keep the current in Eq. (15) conserved, $I_{L}=-I_{R}$, which for symmetric bias occurs if $\Gamma_{L}=\Gamma_{R}$, even without a selfconsistent calculation of $\Delta[25,53,57]$. The density of states in the lead $\alpha$ and the associated tunneling amplitudes $\left|t_{\alpha}(k)\right|^{2}$ are encoded in the quantities $\Gamma_{\alpha}^{ \pm}=2 \pi \sum_{k}\left|t_{\alpha}(k)\right|^{2} \delta\left(E \pm \epsilon_{k \alpha}\right)$, with $-(+)$ for particles (holes). In a realistic device scenario, however, one may have to represent the leads in the site basis and employ a recursive approach to calculate the self-energy [56].

Equation (15) allows a microscopic analysis of the charge transfer through the Kitaev chain, where two processes contribute. The term containing $G_{1, N}^{r}$ describes the usual direct transfer $(D)$ of a quasiparticle from the left to the right lead through a normal conducting system, but here in presence of the $p$-wave superconductivity embodied by $\Delta$. The second term in Eq. (15), i.e., the one including $G_{1, N+1}^{r}$, describes the Andreev reflection-the incoming electron is reflected back as a hole and a right-moving Cooper pair is formed inside the Kitaev chain $[58,59]$. In the third possible process, the right-moving Cooper pair in the chain is formed by an electron coming from the left and a hole coming from the right. 
This process, named crossed Andreev reflection, does not contribute to the current in a symmetric bias configuration. We give the exact analytic form of $G_{1, N}^{r}$ and $G_{1, N+1}^{r}$ in Appendix G in terms of Tetranacci polynomials, see in particular Eqs. (G9) and (G11).

The relative weight of the two contributing processes depends on the chosen parameters of the Kitaev chain $(\mu, t, \Delta)$, as we will see in the context of the zero temperature conductance in the next section.

\section{LINEAR TRANSPORT}

The conductance $G:=\lim _{V \rightarrow 0} \partial I / \partial V$ is easily calculated from Eq. (15). At $T=0 \mathrm{~K}$, one finds the simple formula

$$
\begin{aligned}
G & =\frac{e^{2}}{h}\left\{\Gamma_{L}^{-} \Gamma_{R}^{-}\left|G_{1, N}^{r}\right|^{2}+\Gamma_{L}^{-} \Gamma_{L}^{+}\left|G_{1, N+1}^{r}\right|^{2}\right\}_{E=0} \\
& =: G_{D}+G_{A},
\end{aligned}
$$

accounting for direct transport and Andreev reflection, respectively.

In the following, we make use of the analytic expressions for $G_{1, N}^{r}, G_{1, N+1}^{r}$ derived in Appendix $\mathrm{G}$ to give the closed formulas for $G_{1, N}^{r}, G_{1, N+1}^{r}$ at $E=0$ (derived in Appendix H). For simplicity, we consider the wide band limit, where the tunneling amplitudes $t_{L}, t_{R}$ and the densities of states $\rho_{L}, \rho_{R}$ in the leads are constant. Thus, $\Gamma_{L, R}^{ \pm}=\Gamma_{L, R}=$ const. We find that the Green's functions at $E=0$ are given by

$$
\begin{gathered}
\left.G_{1, N}^{r}\right|_{E=0}=(-1)^{N-1} \frac{p^{N-1}+m^{N-1}}{\left|q_{+}\right|^{2}+\gamma_{L} \gamma_{R}\left(p^{N-1}+m^{N-1}\right)^{2}} q_{-}, \\
\left.G_{1, N+1}^{r}\right|_{E=0}=-i \gamma_{R} \frac{p^{2 N-2}-m^{2 N-2}}{\left|q_{+}\right|^{2}+\gamma_{L} \gamma_{R}\left(p^{N-1}+m^{N-1}\right)^{2}},
\end{gathered}
$$

with $p=t+\Delta, m=t-\Delta$, and $\gamma_{L, R}=\Gamma_{L, R} / 2$. The polynomial $q_{s}(s= \pm 1)$, given by

$$
\begin{aligned}
q_{s}= & p^{N-2}\left[s p^{2} x_{N, 0}+i p x_{N-1,0}\left(s \gamma_{L}-\gamma_{R}\right)\right. \\
& \left.+x_{N-2,0} \gamma_{L} \gamma_{R}\right],
\end{aligned}
$$

carries information on the spectral structure of the isolated Kitaev chain, since the determinant of the isolated Kitaev Hamiltonian with $N$ sites is $(-1)^{N} p^{2 N} x_{N, 0}^{2}$ [39]. The closed form of the term $x_{j, 0}$ for an arbitrary integer $j$ is

$$
x_{j, 0}=\frac{R_{+}^{j+1}-R_{-}^{j+1}}{R_{+}-R_{-}},
$$

with $R_{ \pm}=\left(-\mu \pm \sqrt{\mu^{2}-4 m p}\right) /(2 p)$.

We find for the conductance the closed form

$$
G=\frac{e^{2}}{h} \frac{\gamma_{L} \gamma_{R}\left(p^{N-1}+m^{N-1}\right)^{2}}{\left|q_{+}\right|^{2}+\gamma_{L} \gamma_{R}\left(p^{N-1}+m^{N-1}\right)^{2}} .
$$

The conductance in the limit $N \rightarrow \infty$ takes the value $e^{2} / h$. We also get the value $G=e^{2} / h$ for the linear conductance at the Kitaev points, independent of the value of the coupling strengths $\gamma_{L, R}$, since the terms in $q_{s}$ vanish there. The $G_{L}$ from Eqs. (32) and (33) in Ref. [29] can be obtained from the formulae in Appendix H, setting $\mu=0$ and $\eta=1$ (in Ref. [29] one contact is effectively grounded).
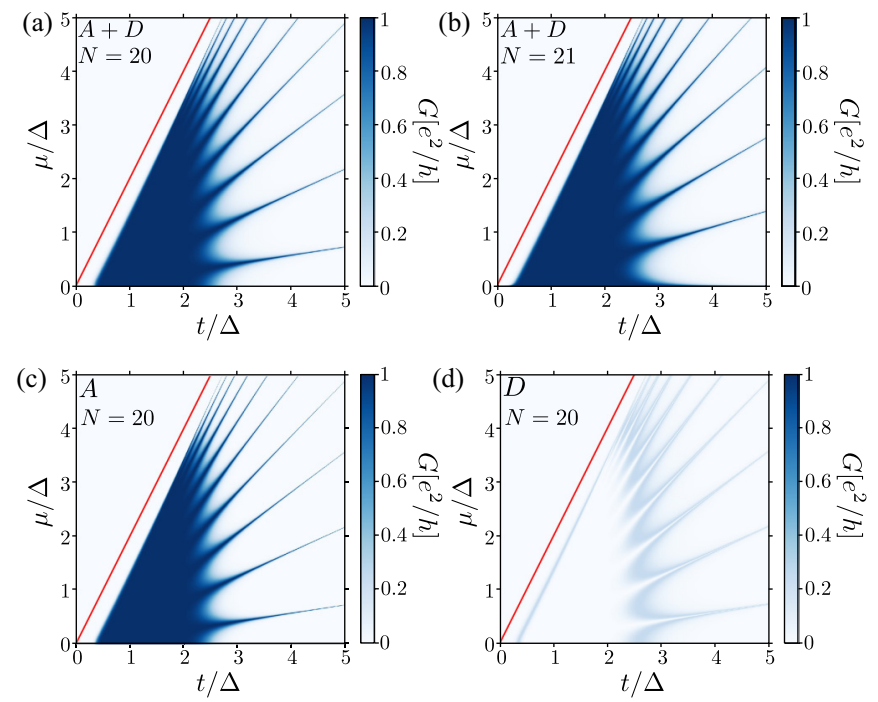

FIG. 5. Conductance $G$ (wide band limit) in units of $e^{2} / h$ for $\gamma_{L, R} / \Delta=0.001$ as function of $\mu / \Delta$ and $t / \Delta$. (a), (b) The roughly triangular plateau of high conductance is bounded (with some padding [39]) by the phase boundary (red line) and branches out into distinct lines when the magnitude of decay length and system length become comparable. Those lines of high conductance follow the Majorana lines given by Eq. (4), with one of them always coinciding with the $\mu=0$ axis for odd $N$. (c) The most important contribution to the conductance $G=G_{A}+G_{D}$ is the Andreev term. (d) The direct term $G_{D}$ only broadens the conductance plateau.

Besides the Kitaev points, the behavior of the conductance is more intricate and depends on the parameters setting. In particular, on the zero-energy Majorana lines of the isolated chain, see Eq. (4), the term $x_{N, 0}$ vanishes, although, due to the coupling to the leads, the whole polynomial $q_{s}$ does not. For the special case of symmetric coupling $\gamma_{L}=\gamma_{R}$, however, the conductance along the Majorana lines becomes nearly independent of the coupling. The behavior of the conductance in the $t / \Delta-\mu / \Delta$ plane is shown in Figs. 5(a) and 5(b) for the case of $N=20$ and $N=21$ sites. While in the vicinity of the Kitaev points the conductance is large and close to $e^{2} / h$, as the ratio of $t / \Delta$ increases it remains so large only in close vicinity of the Majorana lines.

To better understand this behavior, we examine more closely the two contributions to the conductance, $G_{D}$ and $G_{A}$, see Eq. (16). We find

$$
\begin{aligned}
G_{D} & =\frac{e^{2}}{h} \frac{\gamma_{L} \gamma_{R}\left(p^{N-1}+m^{N-1}\right)^{2}}{\left[\left|q_{+}\right|^{2}+\gamma_{L} \gamma_{R}\left(p^{N-1}+m^{N-1}\right)^{2}\right]^{2}}\left|q_{-}\right|^{2}, \\
G_{A} & =\frac{e^{2}}{h} \frac{\gamma_{L}^{2} \gamma_{R}^{2}\left(p^{2 N-2}-m^{2 N-2}\right)^{2}}{\left[\left|q_{+}\right|^{2}+\gamma_{L} \gamma_{R}\left(p^{N-1}+m^{N-1}\right)^{2}\right]^{2}},
\end{aligned}
$$

with $q_{ \pm}$from Eq. (19). For details of the calculation, see Appendix $\mathrm{H}$. The contributions $G_{A}$ and $G_{D}$ for the case $N=20$ are depicted in Figs. 5(c) and 5(d). The difference between the Andreev and the direct term originates from the function $q_{-}$which appears in the numerator of $G_{D}$. For $\gamma_{L, R} \ll \Delta$, the $q_{-}$factor is small as long as $\xi \ll d N$, i.e., inside the triangular conductance plateau. Here $x_{N, 0}$ is exponentially small due to the existence of in-gap states and $x_{N-1,0}, x_{N-2,0}$ are 


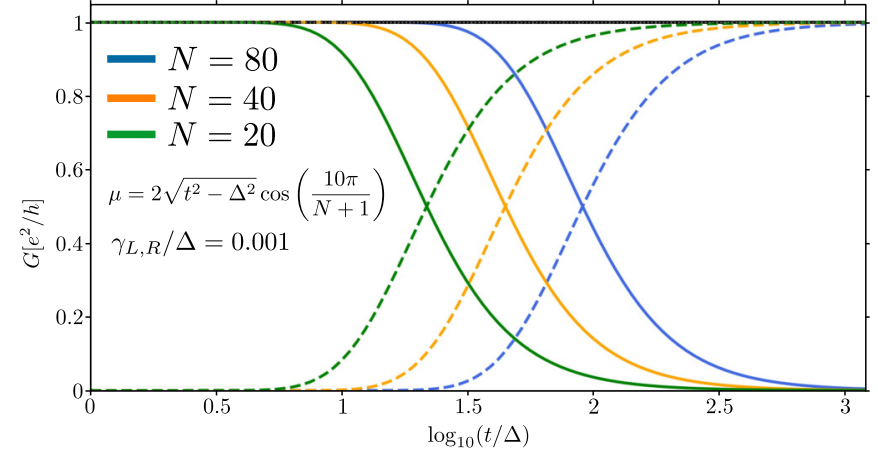

FIG. 6. Conductance contributions $G_{A}$ and $G_{D}$ (wide band limit, $T=0 \mathrm{~K})$ in units of $e^{2} / h$ as function of $t / \Delta$ along the zero energy line, with $\mu$ adjusted to obey Eq. (4) for $n=10$ and with $\gamma_{L}=\gamma_{R}=0.001 \Delta$, for different chain lengths. The Andreev term (solid lines) mostly contributes in the vicinity of the Kitaev point and decreases for larger ratios $t / \Delta$, while $G_{D}$ (dashed lines) shows the opposite behavior. The total conductance (black line) $G=G_{A}+G_{D}$ stays close to $e^{2} / h$, since a contributing zero-energy eigenstate of the isolated Kitaev chain is always available. The Andreev term accounts here for the reflection $R_{D}=1-T_{D}$, where $T_{D}$ is the transmission amplitude of the direct term.

suppressed by $\gamma_{L, R}$. In the region of the plateau, $q_{+}$is also small, thus $G_{A}$ is enhanced while $G_{D}$ is suppressed. In the limit of vanishing order parameter, $\Delta=0$, it immediately follows from the above equations that $G=G_{D}, G_{A}=0$, since-as expected-the Andreev contribution vanishes. For $\xi \gg N d$ and leaving the discrete lines of nonzero conductance aside for a second, we find no in-gap states with zero or even exponentially small energy anymore; the function $q_{s}$ grows for increasing values of $\mu / \Delta$ and/ or $t / \Delta$, which leads to a suppression of both conduction terms $G_{D} \propto\left|q_{-}\right|^{2} /\left|q_{+}\right|^{4}$, $G_{A} \propto 1 /\left|q_{+}\right|^{4}$. For intermediate parameter values $\xi \approx N d$, the polynomials $x_{N-1,0}, x_{N-2,0}$ become important. They describe essentially the spectrum of a Kitaev chain with $N-1, N-2$ sites, i.e., $\xi \gtrsim(N-j) d$ for $j=1,2$. Their contributions define the crossover region between the triangular plateau of high conductance and the region featuring separated Majorana lines within the topologically nontrivial phase when $\xi \gg N d$. Note that the crossover region is influenced by $\gamma_{L, R}$ too.

Let us turn to the conductance along the Majorana lines, given by Eq. (4). On those lines, the function $x_{N, 0}$ vanishes and thus the functions $q_{s}$ have minima in $\mu$. The value of $q_{s}$ varies strongly around these minima and leads to the appearance of low conductance regions between the Majorana lines for $\xi \gg N d$. The ratio of $G_{D}$ and $G_{A}$ changes along those lines as depicted in Fig. 6 starting with $G_{A}=1$ and $G_{D}=0$ at Kitaev points and converges to $G_{A}=0$ for $t / \Delta \rightarrow \infty$. This behavior is independent of the chosen line. Remarkably, the sum $G_{D}+G_{A}$ is seemingly constant and equal to $e^{2} / h$; it is, in fact, very slightly suppressed due to Eq. (21), becoming fully quantized only in the thermodynamic limit.

\section{NONLINEAR TRANSPORT}

The nonlinear transport effects are captured by the differential conductance $\partial I / \partial V$. At $T=0 \mathrm{~K}$ and using Eq. (15) we
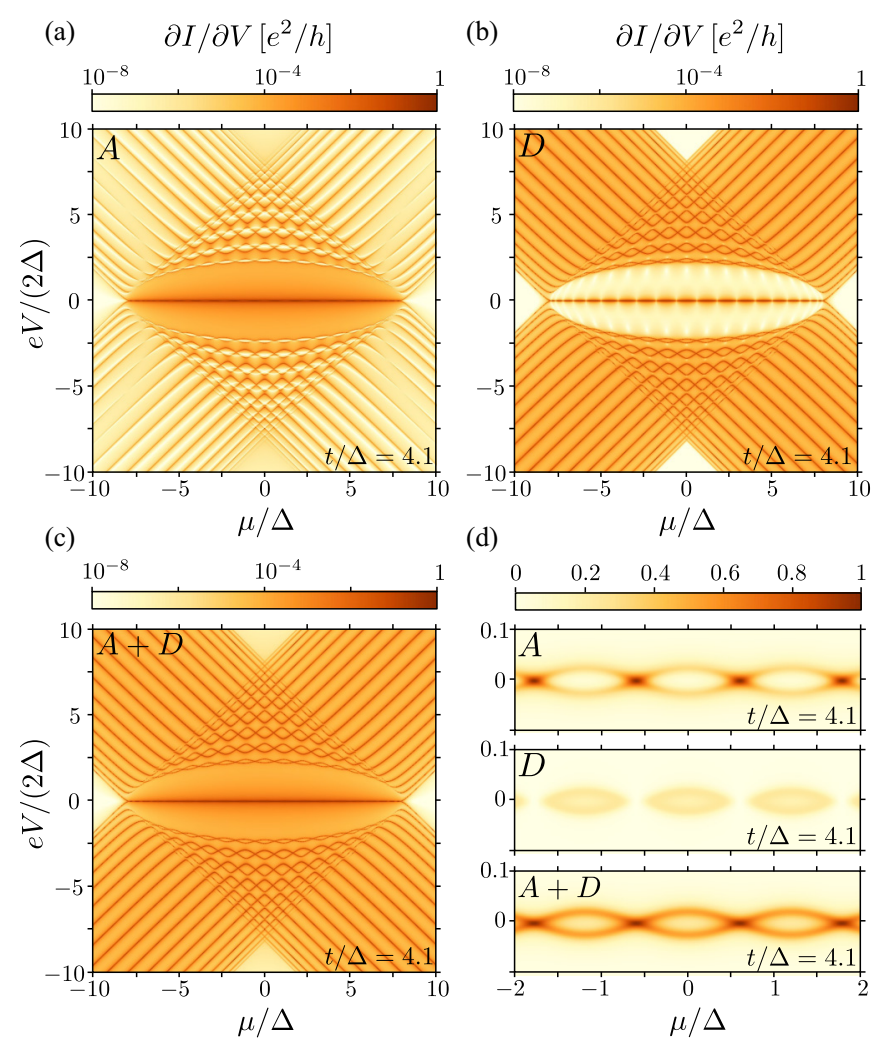

FIG. 7. Differential conductance as a function of $e V /(2 \Delta)$ and $\mu / \Delta$ for $|t / \Delta|=4.1, \gamma_{L}=\gamma_{R}=0.02 \Delta$, and $N=20$. (a) The Andreev term is the dominant contribution to the differential conductance for the in-gap states but also affects the excitations. (b) The direct contribution to $\partial I / \partial V$ is present for all eigenstates; its strength inside the gap depends on the parameters. (c) The total differential conductance is the sum of the Andreev and the direct terms. (d) The dark stripe for $V \approx 0$ of the Andreev term in (a) has, in fact, a braidlike structure, since the chain is too short $(N d \sim 4 \xi)$ to support zero-energy eigenstates everywhere $[4,28,39]$. The Andreev reflections are stronger around the values of $\mu$ where exact zero-energy states are present. Direct process contributions enhance the transport between two Andreev peaks.

find

$$
\frac{\partial I}{\partial V}=\frac{e^{2}}{2 h} \sum_{E= \pm V / 2} \Gamma_{L}^{-}\left(\Gamma_{R}^{-}\left|G_{1, N}^{r}\right|^{2}+\Gamma_{L}^{+}\left|G_{1, N+1}^{r}\right|^{2}\right)
$$

where we set $V_{L}=-V_{R}=V / 2$. We depicted $\partial I / \partial V$ and its Andreev $(A)$ and direct $(D)$ contributions given by the $G_{1, N+1}^{r}$ $\left(G_{1, N}^{r}\right)$ terms in Figs. 7 and 8.

As expected, the Andreev term is slightly smaller than $e^{2} / h$ around $V \approx 0$ for $|\mu|<2|t|$ and $\xi /(N d) \ll 1$, while the direct term is weak. Outside $V \approx 0$, the roles of Andreev and direct contributions are exchanged, though the Andreev term reemerges, contributing a significant fraction of a conductance quantum at the resonances with the quasiparticle energy levels and inside the avoided crossings between higher excitations; there the involved eigenstates of the Kitaev chain have again significant contributions from both particle and hole sectors (cf. Fig. 2).

A special situation arises at the Kitaev points, where $\mu=$ 0 and $|t|=|\Delta|$. Here the isolated Kitaev chain hosts only 


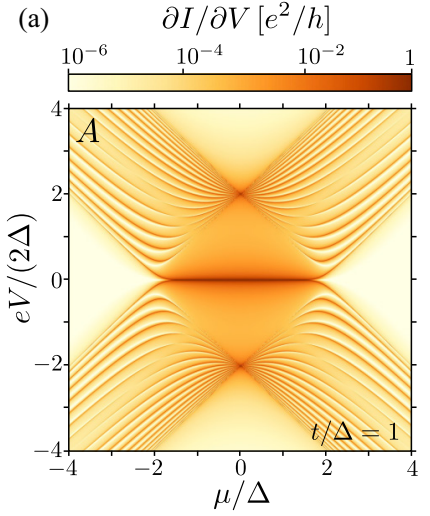

(b)
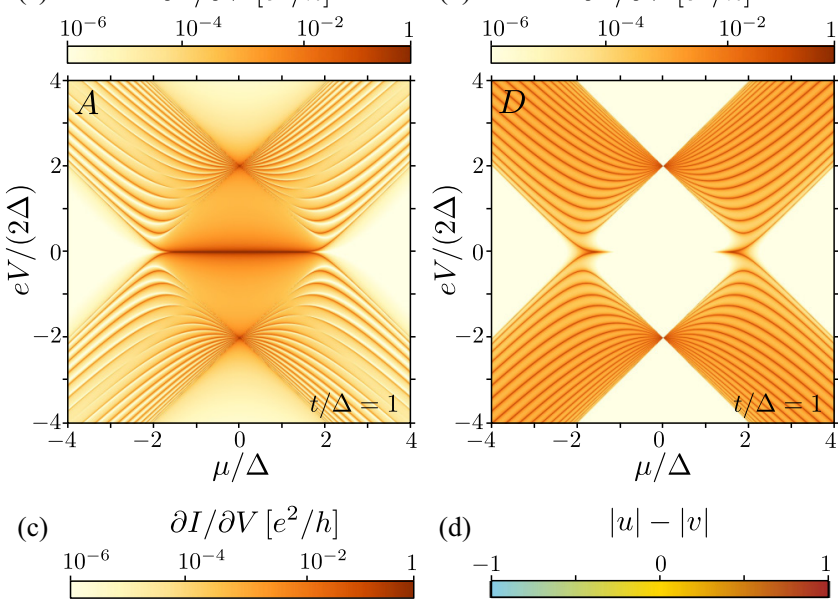

(d)

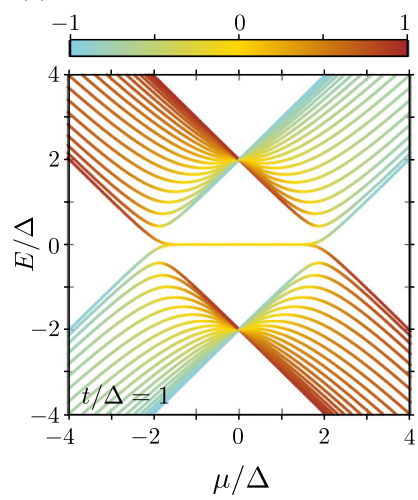

(a)

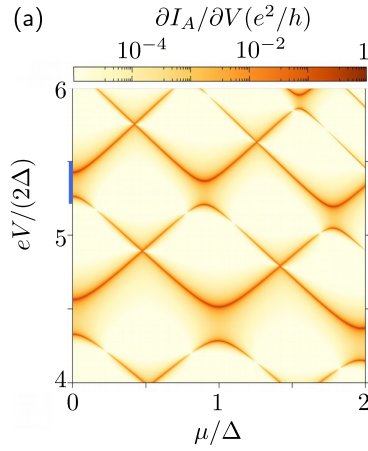

(b)

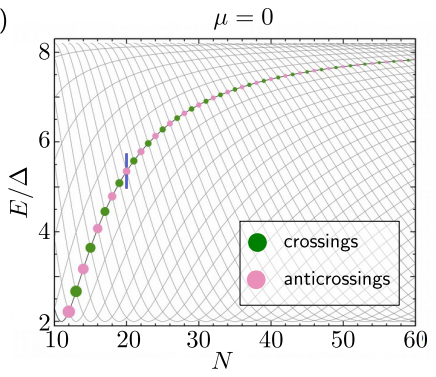

(c)
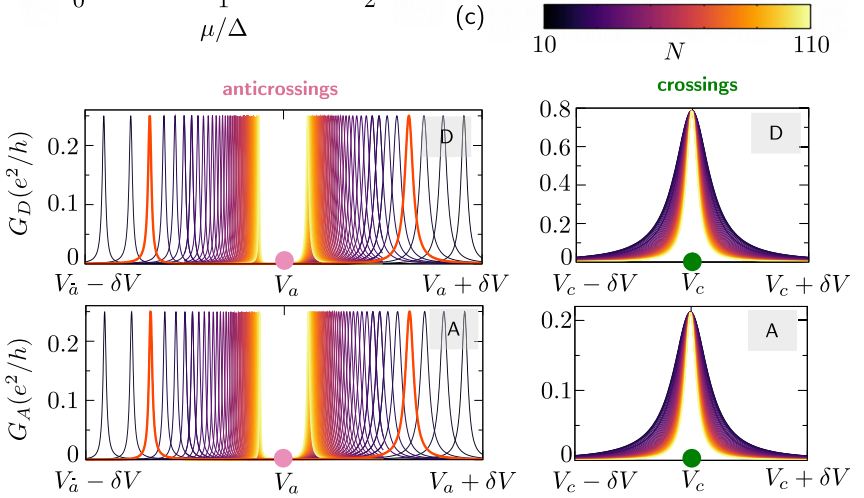

FIG. 9. Differential conductance as a function of $e V /(2 \Delta)$ for $t / \Delta=4.1$ and $\gamma_{L}=\gamma_{R}=0.02 \Delta$. (a) A typical pattern arising in the Andreev term as a function of $\mu / \Delta$, here for $N=20$ [zoom into Fig. 7(a)]. The blue dashes in (a) and (b) mark the bias range from which the red traces in (c) are taken. (b) Grey lines in the background trace $E[n \pi /(N+1)]$, with $n \in[0, N]$. The positions of exact and avoided crossings along $E\left[\kappa_{1}=6 \pi /(N+1)\right]$ are marked with green and pink points, respectively. (c) The two contributions $G_{D}=\partial I_{D} / \partial V$ and $G_{A}=\partial I_{A} / \partial V$ at $\mu=0$ in a small bias window around the center $V_{c}\left(V_{a}\right)$ of the (anti)crossing. The bias window width and sampling are chosen adaptively; the curves are rescaled horizontally so as to show them clearly also for large $N$. The curves highlighted in red at the anticrossings are the $\partial I_{D} / \partial V$ traces at $N=20$ indicated in (a) and (b).

$\mu$ values where the MZM are present [36-39], i.e., at the zero-energy crossings. In between these specific parameter values, the importance of the Andreev contribution decreases and the direct term starts to contribute.

Increasing the length of the Kitaev chain increases the separation between the left and right Majorana components of the topological state. The subgap conductance becomes dominated by the Andreev processes, while the importance of direct transmission diminishes. In the high bias differential conductance, the surprisingly strong revival of Andreev processes turns out to be independent of the length and reaching $e^{2} /(4 h)$. A typical pattern of exact and avoided crossings in the Andreev contribution as a function of $\mu$ and $V$ is shown for $N=20$ in Fig. 9(a). To track the length dependence of the Andreev peaks, we examine the neighborhood of the crossings and anticrossings in the excitation spectrum, where either the particle/hole mixing is maximal (anticrossings) or there are two degenerate states of opposite particle/hole character available (crossings; see also Fig. 2). We set $\mu=0$ and track the bias windows around $E\left(\kappa_{1}\right)=6 \pi /(N+1)$ [which fixes $\left.\kappa_{2}=(N-5) \pi /(N+1)\right]$. For even $N, E\left(\kappa_{1}\right)$ will give 
us the position of the anticrossing centers, for odd $N$-of the crossings. The evolution of $E\left(\kappa_{1}\right)$ with $N$ is shown in Fig. 9(b). We scan the differential conductance in a bias window around the centers of these (anti)crossings, with the width and sampling of the bias window chosen adaptively, accounting for denser peaks at high $V$ and $N$. The resulting differential conductance curves for each $N \in[10,110]$ are shown in Fig. 9(c), centered on the bias corresponding to the appropriate center of the (anti)crossing. The peak amplitudes for both direct and Andreev contributions are length independent, although the peak width in both cases decreases with the chain length. At the anticrossings, where the particle and hole sectors are fully mixed, the amplitude of direct and Andreev terms are the same, equal to $0.25 e^{2} / h$. In the case of crossings, the reappearance of the Andreev contribution is due to the presence of two degenerate states, from which mixed particle-hole solutions can be constructed, although the two components do not have equal weight. The peak values now differ, with the Andreev contribution at $\sim 0.21 e^{2} / h$ and the direct contribution at $\sim 0.79 e^{2} / h$. The Andreev contribution is suppressed with increasing $t / \Delta$, in a way analogous to the zero-bias conductance (cf. Fig. 6).

\section{CONCLUSIONS}

In this paper, we have investigated linear and nonlinear transport across a finite Kitaev chain in an N-S-N setup, with symmetrically applied bias. Using the analytical methods developed to study the spectrum of the isolated Kitaev chain [39], we could provide closed formulas for the relevant Green's functions and, in turn, for the linear and differential conductance at zero temperature. We have analyzed the quasiparticle spectrum with its complex pattern of exact and avoided crossings being governed by the inversion symmetry and related this pattern to the calculated transport spectra. Perhaps counterintuitively, our results show that direct transmission processes also contribute to the subgap transport mediated by the topological states and, likewise, that the Andreev processes participate in the transport at high bias, especially when the involved states are nearly equal superpositions of particle and hole solutions. At the anticrossing, the direct transmission and Andreev processes contribute equally. Furthermore, the two split peaks reach the maximum value of $0.5 e^{2} / h$, independent of the chain length. Regarding the linear conductance, the maximum value of $e^{2} / h$ is reached only near the Kitaev points.

In summary, our work provides a complete analytical description of transport through an archetypal topological superconductor, the Kitaev chain, extending our knowledge of this system beyond what can be gleaned from minimal models reduced to topological states alone. Since some of the observed spectral and transport features (such as the exact and avoided crossings in the higher excitation spectrum) are generic to $1 \mathrm{D}$ topological superconductors, our complete analytical and numerical treatment can provide a valuable benchmark and insight for the study of other model systems, such as, for example, the one based on $s$-wave proximitized Rashba nanowires.
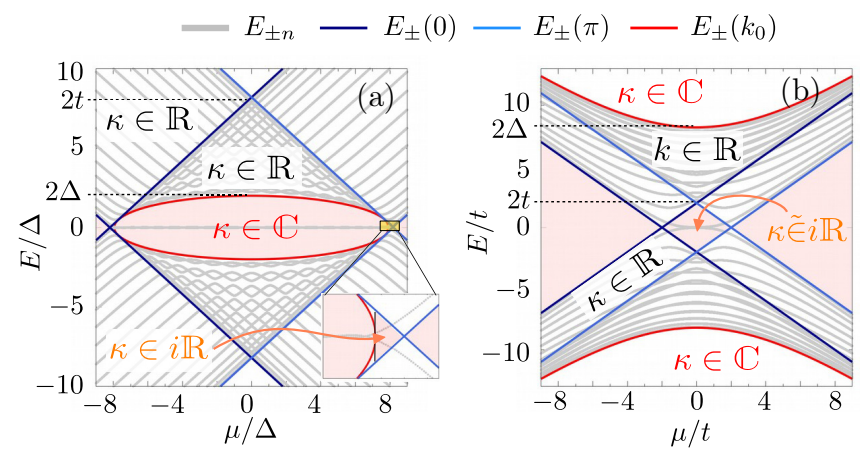

FIG. 10. The band extrema of a bulk Kitaev chain as a function of $\mu$, for (a) $t=4.1 \Delta$ and (b) $\Delta=4.1 t$. The numerical energy levels for a chain with $N=20$ sites are shown in grey for comparison. The types of allowed solutions for the wave number $\kappa$ in a finite chain are indicated. Purely imaginary $\kappa$ are allowed in the small range of $\mu$ indicated in the inset of (a). The notation $\kappa \tilde{\in} i \mathbb{R}$ in the topological range of $\mu$ in (b) means that the allowed wave vectors can also have a constant real part, $\pi / 2$.

\section{ACKNOWLEDGMENTS}

N.L. and B.M. thank for the financial support of the Elite Netzwerk Bayern via the IGK Topological Insulators and the Deutsche Forschungsgemeinschaft via SFB Project No. B04. B.M. would like to acknowledge funding from the Science and Engineering Research Board (SERB), Government of India under Grant No. STR/2019/000030, and the Ministry of Human Resource Development (MHRD), Grant No. STARS/APR2019/NS/226/FS under the STARS scheme and the Young faculty research fellowship from the Ministry of Electronics and Information Technology (MEITY), Government of India, implemented by Digital India Corporation (formerly Media Lab Asia).

\section{APPENDIX A: BULK GAP}

The bulk gap in the Kitaev spectrum is easily estimated from the dispersion relation Eq. (2). The condition for the vanishing first derivative at the band extrema is fulfilled at three values of bulk momentum $k$ :

$$
k_{1}=0, \quad k_{2}=\pi, \quad k_{0}=\arccos \left(\frac{\mu t}{2\left(\Delta^{2}-t^{2}\right)}\right) .
$$

Examples of the spectra of the Kitaev chain with $N=20$ sites as a function of $\mu$, together with the lines denoting the bulk energies at the band extrema, are shown in Fig. 10. The wave functions in a finite Kitaev chain can be described by purely real, purely imaginary, or complex wave vectors $\kappa$, in the regions marked in the figure. The bulk gap is marked with light red shading.

\section{APPENDIX B: SELECTED QUANTUM BASES FOR THE KITAEV CHAIN}

The operators associated with the Kitaev chain can be represented in several bases, each suited to facilitate some specific calculation. We give here an overview of the four 


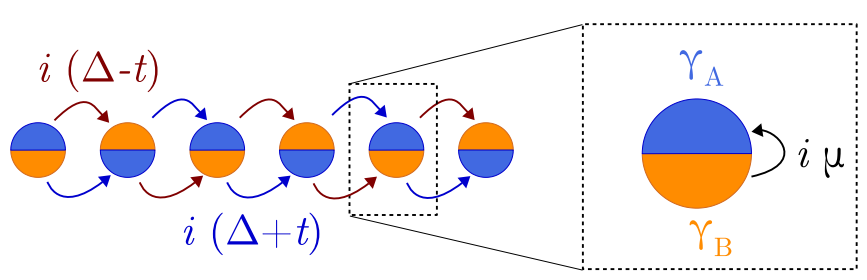

FIG. 11. Kitaev chain described through the Majorana operators. The effective hoppings are all imaginary, with $i(\Delta \pm t)$ describing the hopping between two sites and the $i \mu$ relating the Majorana $A$ and $B$ sublattices on one site.

basis choices which will be used in these Appendices, together with the rationale behind this choice.

(1) Default Bogoliubov-de Gennes basis, used throughout the main text and given by

$$
\hat{\Psi}=\left(d_{1}, \ldots, d_{N}, d_{1}^{\dagger}, \ldots, d_{N}^{\dagger}\right)^{T} .
$$

This basis neatly separates particle and hole sectors of the system. The representations of the physical quantities in this basis do not carry any labels: the Green's functions are $G^{s}$, where $s=<,>, a, r$, the self-energies $\Sigma_{\alpha}^{s}$, the $\Gamma$ matrices $\Gamma_{\alpha}$, and $\alpha=L, R$.

(2) Chiral basis is defined by

$$
\hat{\Psi}_{c}=\left(\gamma_{1}^{A}, \ldots, \gamma_{N}^{A}, \gamma_{1}^{B}, \ldots, \gamma_{N}^{B}\right)^{T}
$$

where $\gamma^{A / B}$ are the Majorana operators, given by $\gamma_{j}^{A}=\left(d_{j}+\right.$ $\left.d_{j}^{\dagger}\right) / \sqrt{2}, \gamma_{j}^{B}=i\left(d_{j}^{\dagger}-d_{j}\right) / \sqrt{2}$. The Hamiltonian terms of the Kitaev chain in this basis are illustrated in Fig. 11. We name it chiral because in this basis the chiral symmetry has an especially simple representation, $\mathcal{C}=\sigma_{z} \otimes \mathbb{1}_{N \times N}$. The physical quantities in this representation are denoted by the subscript $c$, e.g., $\mathcal{H}_{c}, I_{c}$. We use it only for the spectrum calculations in Appendix D to highlight how the $A$ components of an eigenstate are mapped by inversion onto its $B$ components.

(3) Site-ordered particle-hole basis is just a rearranged default basis, with

$$
\hat{\tilde{\Psi}}=\left(d_{1}, d_{1}^{\dagger}, \ldots, d_{N}, d_{N}^{\dagger}\right)^{T} .
$$

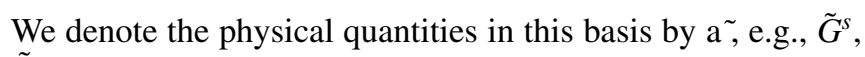
$\tilde{\Sigma}_{\alpha}$. The transformation between this and the default basis is given by $\hat{\tilde{\Psi}}=U \hat{\Psi}$, with

$$
U_{n m}= \begin{cases}\delta_{m,(n+1) / 2} & \text { for } n \text { odd } \\ \delta_{m, N+n / 2} & \text { for } n \text { even, }\end{cases}
$$

with $n, m=1, \ldots, 2 N$. Since $U$ is just a permutation matrix, an observable $A$ transforms as $\tilde{A}=U A U^{T}$. This is our intermediate basis in Appendix E, in which the site-specific Green's functions are expressed most conveniently.

(4) Site-ordered Majorana basis is a rearranged chiral basis, with

$$
\hat{\Psi}_{M}=\left(\gamma_{1}^{A}, \gamma_{1}^{B}, \ldots, \gamma_{N}^{A}, \gamma_{N}^{B}\right)
$$

The physical quantities in this basis are denoted by the subscript ${ }_{M}$. The unitary transformation to the default basis, such that $\hat{\Psi}_{\mathrm{M}}=\mathrm{T} \hat{\Psi}$, is given by the matrix $\mathrm{T}$

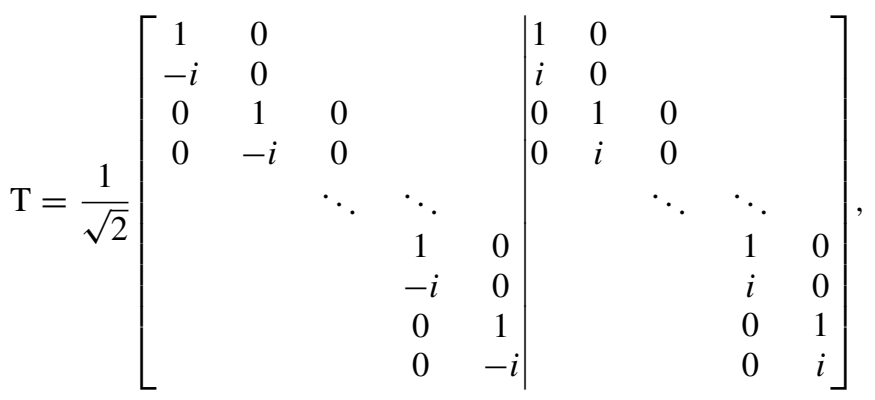

where | separates the first $N$ and the last $N$ columns. The physical quantities transform as $A_{M}=\mathrm{T}_{A} \mathrm{~T}^{\dagger}$. We use this basis to set up the polynomial sequences in Appendix C. The same type of sequences define the eigenvectors of the system in Appendix D. In Appendix G, we take advantage of the block-tridiagonal form of the Hamiltonian in this basis (the elements of the Hamiltonian are illustrated in Fig. 11).

\section{APPENDIX C: TETRANACCI POLYNOMIALS AND THEIR CLOSED FORMULA}

The eigenvectors of Hamiltonians describing the nearestneighbor hopping in a Su-Schrieffer-Heeger (SSH) chain could be obtained using the Fibonacci polynomials [39], where each term in the polynomial sequence is determined by its immediate neighbors. The presence of further nondiagonal terms in the Hamiltonian will result in more complex polynomial sequences, including more elements in the recursion formula. In the general Kitaev chain, which is equivalent to two SSH-like chains additionally coupled by $\mu$, we must use polynomial sequences where each term is determined by four preceding ones. These Tetranacci polynomials are essential for the calculations of the excited-state wave functions in Appendix $\mathrm{D}$ and for the exact calculation of the Green's functions in Appendix G.

\section{Definition and basic properties}

The eigenvectors of a Kitaev chain can be expressed through two sequences of Tetranacci polynomials, one for the $A$ and one for the $B$ elements of the eigenvectors (later referred to as $\vec{v}_{A}$ and $\vec{v}_{B}$ ). We describe this procedure in Appendix D.

The characteristic polynomial of the isolated Kitaev chain, which we will need for the calculation of the Green's functions, can be expressed through four Tetranacci sequences $x_{j}, y_{j}, \chi_{j}$, and $\mathcal{Y}_{j}$, which obey two equivalent sets of coupled equations. The first set is

$$
\begin{aligned}
x_{j+1} & =\frac{-i \mu}{b} x_{j}+\frac{a}{b} x_{j-1}+\frac{E}{b} y_{j}, \\
\chi_{j+1} & =\frac{-i \mu}{b} \chi_{j}+\frac{a}{b} \chi_{j-1}+\frac{E}{b} \mathcal{Y}_{j}, \\
y_{j+1} & =\frac{i \mu}{a} y_{j}+\frac{b}{a} y_{j-1}+\frac{E}{a} x_{j}, \\
\mathcal{y}_{j+1} & =\frac{i \mu}{a} \mathcal{Y}_{j}+\frac{b}{a} \mathcal{Y}_{j-1}+\frac{E}{a} \chi_{j},
\end{aligned}
$$

and the second set reads

$$
x_{j+1}=\frac{-i \mu}{b} x_{j}+\frac{a}{b} x_{j-1}+\frac{E}{a} \chi_{j},
$$


TABLE I. The first values of the Tetranacci polynomials $x_{j}, y_{j}$, $\chi_{j}$, and $\mathcal{Y}_{j}$

\begin{tabular}{ccccc}
\hline \hline$j$ & $x_{j}$ & $\mathcal{Y}_{j}$ & $\chi_{j}$ & $y_{j}$ \\
\hline-3 & $i \mu b / a^{2}$ & $-i \mu a / b^{2}$ & $-E / b$ & $-E / a$ \\
-2 & $b / a$ & $a / b$ & 0 & 0 \\
-1 & 0 & 0 & 0 & 0 \\
0 & 1 & 1 & 0 & 0 \\
1 & $-i \mu / b$ & $i \mu / a$ & $E / b$ & $E / a$ \\
\hline \hline
\end{tabular}

$$
\begin{aligned}
\chi_{j+1} & =\frac{i \mu}{a} \chi_{j}+\frac{b}{a} \chi_{j-1}+\frac{E}{b} x_{j}, \\
y_{j+1} & =\frac{-i \mu}{b} y_{j}+\frac{a}{b} y_{j-1}+\frac{E}{a} \mathcal{Y}_{j}, \\
\mathcal{Y}_{j+1} & =\frac{i \mu}{a} \mathcal{Y}_{j}+\frac{b}{a} \mathcal{Y}_{j-1}+\frac{E}{b} y_{j} .
\end{aligned}
$$

Using the relationships defined by these equations, we can decouple the four sequences and find that each of the $x_{j}, y_{j}$, $\chi_{j}, \mathcal{Y}_{j}$ polynomials obeys the same recursion formula as $x_{j}$ (and as the eigenvector entries $\vec{v}_{A}, \vec{v}_{B}$ ),

$$
\begin{aligned}
x_{j+2}= & \frac{E^{2}+a^{2}+b^{2}-\mu^{2}}{a b} x_{j}-x_{j-2} \\
& +i \mu \frac{b-a}{a b}\left(x_{j-1}+x_{j+1}\right) .
\end{aligned}
$$

The four sequences differ only in their initial values, given in Table I. They appear at first as separated objects but they are in fact connected by a symmetry relation. By exchanging all $a$ terms by $b$ terms and vice versa and turning the sign of $\mu$ into $-\mu, x_{j}\left(\chi_{j}\right)$ transforms into $y_{j}\left(\mathcal{Y}_{j}\right)$.

Although Eqs. (C1) and (C2) are equivalent to Eq. (C3), each description has its own advantages, often unseen in the other. For example, the comparison of Eq. (C1d) with Eq. (C2d) yields

$$
b \chi_{j}=a y_{j},
$$

and more such relationships can be found.

Note also that Eq. (C3) is invariant under the inversion symmetry and exchange of $\Delta \rightarrow-\Delta$. Further, all four polynomials carry no physical unit and $x_{j}, \mathcal{Y}_{j}\left(\chi_{j}, y_{j}\right)$ are real (pure imaginary) objects.

However, the most important reason to present Eqs. (C1) and (C2) is the limiting case of $E=0$, which we need to obtain the conductance formula at zero bias and temperature later. While in Eq. (C3) seemingly not much happens at $E=$ 0 , in Eqs. (C1) and (C2) we find that $x_{j}, y_{j}, \chi_{j}, \mathcal{Y}_{j}$ become decoupled and obey simplified recursion formulas. We denote the polynomials in the case of $E=0$ with $x_{j, 0}, y_{j, 0}, \chi_{j, 0}, \mathcal{Y}_{j, 0}$. The polynomials $\chi_{j, 0}=y_{j, 0} \equiv 0$ due to the initial values in Table I, and $x_{j, 0}$ and $\mathcal{y}_{j, 0}$ reduce to Fibonacci polynomials [60-62]

$$
\begin{aligned}
& b x_{j+1,0}=-i \mu x_{j, 0}+a x_{j-1,0}, \\
& a \mathcal{Y}_{j+1,0}=i \mu \mathcal{Y}_{j, 0}+b \mathcal{Y}_{j-1,0} .
\end{aligned}
$$

A power law ansatz for $x_{j, 0} \propto R^{j}\left(\mathcal{Y}_{j, 0} \propto \tilde{R}^{j}\right)$ leads first to the values of $R_{ \pm}\left(\tilde{R}_{ \pm}\right)$:

$$
R_{ \pm}=\frac{-i \mu \pm \sqrt{4 a b-\mu^{2}}}{2 b}, \quad \tilde{R}_{ \pm}=\frac{i \mu \pm \sqrt{4 a b-\mu^{2}}}{2 a} .
$$

A superposition of $R_{ \pm}^{j}\left(\tilde{R}_{ \pm}^{j}\right)$ leads to

$$
\begin{aligned}
& x_{j, 0}=\frac{R_{+}^{j+1}-R_{-}^{j+1}}{R_{+}-R_{-}}, \\
& \mathcal{Y}_{j, 0}=\frac{\tilde{R}_{+}^{j+1}-\tilde{R}_{-}^{j+1}}{\tilde{R}_{+}-\tilde{R}_{-}} .
\end{aligned}
$$

This closed formula for a Fibonacci polynomial is the socalled Binet form [60-62]. The similarity between $R_{ \pm}$and $\tilde{R}_{ \pm}$ allows us to determine $\mathcal{Y}_{j, 0}$ in terms of $x_{j, 0}$,

$$
\mathcal{Y}_{j, 0}=\left(-\frac{b}{a}\right)^{j} x_{j, 0},
$$

which leads to many simplifications for the conductance formula later.

Please notice that the case of $E=0$ in Eq. (C3) always leads to Fibonacci polynomials even in problems distinct from the Kitaev chain, where Eqs. (C1) and (C2) are unknown.

A second limiting case exists for $\mu=0$. We see directly from Eq. (C3), but not from Eqs. (C1) and (C2), that $x_{j}, y_{j}, \chi_{j}$, $\mathcal{Y}_{j}$ show again a Fibonacci character, only of a different kind compared with the $E=0$ case. Define $u_{j}:=x_{2 j}\left(v_{j}:=x_{2 j-1}\right)$ and thus $u_{j}\left(v_{j}\right)$ obeys

$$
u_{j+1}=\frac{E^{2}+a^{2}+b^{2}}{a b} u_{j}-u_{j-1},
$$

which mimics the form of Eq. (C5) with different coefficients and a power-law ansatz gives their closed form [39].

From the physical point of view, the two sequences of polynomials $u_{j+1}$ and $v_{j+1}$ construct the Green's functions of the $\mu=0$ case, in which the Kitaev chain can be considered as two decoupled SSH-like chains [39].

\section{The closed form of Tetranacci polynomials and their Fibonacci decomposition}

We turn now to the closed formula for any Tetranacci polynomial. Both the characteristic polynomial of the isolated Kitaev chain and its eigenvectors can be written in the form of Tetranacci polynomials. In the context of Green's functions of a Kitaev chain between two leads, the relevant Tetranacci polynomials are $d_{j}^{x}, d_{j}^{y}, d_{j}^{\chi}, d_{j}^{\mathcal{Y}}$ discussed further in Appendix G. We shall therefore derive a closed form for $\xi_{j}$, a general sequence of Tetranacci polynomials obeying Eq. (C3), with arbitrary initial values $\xi_{-2}, \xi_{-1}, \xi_{0}$, and $\xi_{1}$. The expressions for $x_{j}, y_{j}, \chi_{j}, \mathcal{Y}_{j}, d_{j}^{x}, d_{j}^{y}, d_{j}^{\chi}$, and $d_{j}^{\mathcal{Y}}$ can then be obtained by inserting appropriate initial values into the formula for $\xi_{j}$.

The idea is to use a power-law ansatz $\xi_{j} \propto r^{j}(r \neq 0)$ as we did in the limiting cases $E=0$ and $\mu=0$ before. We are left to find all zeros of

$$
r^{4}-\zeta r^{2}+1-\eta\left(r+r^{3}\right)=0
$$


where we used a shorthand notation for the coefficients in Eq. (C3):

$$
\begin{gathered}
\zeta:=\frac{E^{2}+a^{2}+b^{2}-\mu^{2}}{a b}, \\
\eta:=i \mu \frac{b-a}{a b} .
\end{gathered}
$$

One can solve for the zeros by dividing Eq. (C11) by $r^{2}$ and calling $S=r+1 / r$. Thus, we have

$$
S^{2}-2-\zeta-\eta S=0,
$$

and the solutions for $S$ read

$$
S_{1,2}=\frac{\eta \pm \sqrt{\eta^{2}+4(\zeta+2)}}{2} .
$$

Finally, we can get the zeros from $S_{1,2}$. They read

$$
r_{ \pm i}=\frac{S_{i} \pm \sqrt{S_{i}^{2}-4}}{2}, \quad i=1,2 .
$$

An expression for the $S_{1,2}$ in terms of two wave numbers $\kappa_{1}, \kappa_{2}$,

$$
S_{1,2}=2 \cos \left(\kappa_{1,2}\right),
$$

yields directly the physical interpretation of $r_{ \pm i}^{j}$ as plane waves, $r_{ \pm i}^{j}=\exp \left( \pm i \kappa_{i} j\right)$. Since $S_{1,2}$ contains the energy $E$ [via $\zeta$, see Eq. (C13)], it connects the energy $E$ and wave numbers $\kappa_{1,2} \in \mathbb{C}$. Indeed, $S_{1,2}=2 \cos \left(\kappa_{1,2}\right)$ is the shortest form of the dispersion relation of the Kitaev chain in Eq. (2) and implies directly $E\left(\kappa_{1}\right)=E\left(\kappa_{2}\right)$. Note that $\kappa_{1,2}$ are not quantized so far. The details of the connection between $r_{ \pm i}$, $S_{i}$ and the dispersion relation of the isolated Kitaev chain, the wave vectors, and their quantisation rule is given in Ref. [39].

The ansatz for $\xi_{j}$ is simply

$$
\xi_{j}=c_{1} r_{+1}^{j}+c_{2} r_{-1}^{j}+c_{3} r_{+2}^{j}+c_{4} r_{-2}^{j},
$$

and the coefficients $c_{1}, \ldots, c_{4}$ are fixed by $\xi_{-2}, \ldots, \xi_{1}$. Once the $c_{1}, \ldots, c_{4}$ are known in terms of $\xi_{-2}, \ldots, \xi_{1}$, one can reorder Eq. (C17) according to the independent contributions of the initial values. This results in

$$
\xi_{j}=\sum_{i=-2}^{1} \xi_{i} X_{i}(j),
$$

where the functions $X_{i}(j)$ depend only on various powers of $r_{ \pm 1}, r_{ \pm 2}$, see Eq. (C22)-(C24) below, but not on the values of $\xi_{-2}, \ldots, \xi_{1}$. Hence, changing the values of $\xi_{-2}, \ldots, \xi_{1}$ does not change the functions $X_{i}(j)$. As one sees directly from Eq. (C18), there are constraints on $X_{i}(j)$, namely,

$$
X_{i}(j)=\delta_{i, j}, \quad \text { for } i, j=-2, \ldots, 1,
$$

to ensure that the initial values are assumed by $\xi_{j}$. One can understand Eq. (C18) as the counterpart to the Binet form, which is used to determine the closed form expression of Fibonacci polynomials [60-62] [see, e.g., Eq (C8)].

Despite the short form of $\xi_{j}$ in Eq. (C18), the formulas for $X_{i}(j)$ tend to be lengthy, such that we first introduce a shorthand notation for their main pieces. We define the functions $F_{1,2}(j)$ as

$$
\begin{aligned}
& F_{1}(j):=\frac{r_{+1}^{j}-r_{-1}^{j}}{r_{+1}-r_{-1}}=\frac{r_{+1}^{j}-r_{+1}^{-j}}{r_{+1}-r_{+1}^{-1}}, \\
& F_{2}(j):=\frac{r_{+2}^{j}-r_{-2}^{j}}{r_{+2}-r_{-2}}=\frac{r_{+2}^{j}-r_{+2}^{-j}}{r_{+2}-r_{+2}^{-1}},
\end{aligned}
$$

where the right-hand side of both equalities arise due to $r_{i} r_{-i}=1$ for $i=1,2$. Please notice that already $F_{1,2}(j)$ are special solutions of Eq. (C3), since they are constructed in terms of the solutions $r_{ \pm i}$ [see Eq. (D17)].

The polynomials $X_{i}(j)$ read

$$
\begin{aligned}
X_{-2}(j)= & \frac{F_{2}(j)-F_{1}(j)}{S_{1}-S_{2}}, \\
X_{-1}(j)= & \sum_{\sigma=1}^{2} \frac{F_{\sigma}(j+2)+F_{\sigma}(j-1) F_{\bar{\sigma}}(2)-F_{\sigma}(3) F_{\bar{\sigma}}(j)}{\left(S_{1}-S_{2}\right)^{2}}, \\
X_{0}(j)= & \sum_{\sigma=1}^{2} \frac{F_{\sigma}(j+1) F_{\bar{\sigma}}(3)-F_{\sigma}(j+2) F_{\bar{\sigma}}(2)}{\left(S_{1}-S_{2}\right)^{2}} \\
& -\sum_{\sigma=1}^{2} \frac{F_{\sigma}(j-1)}{\left(S_{1}-S_{2}\right)^{2}}, \\
X_{1}(j)= & \sum_{\sigma=1}^{2} \frac{F_{\sigma}(j+2)+F_{\sigma}(j)-F_{\sigma}(j+1) F_{\bar{\sigma}}(2)}{\left(S_{1}-S_{2}\right)^{2}}, \text { (C25) }
\end{aligned}
$$

where $\bar{\sigma}$ is meant as not $\sigma$, e.g., if $\sigma=1$ then we have $\bar{\sigma}=2$ and vice versa. As one sees, the functions $X_{i}(j)$ are a superposition of the solutions $F_{1,2}(j-x)(x=-2,-1,0,1)$, where the coefficients are sometimes $F_{1,2}(2)$ or $F_{1,2}(3)$. Thus, the $X_{i}(j)$ are Tetranacci polynomials as well. As we saw in Eq. (C17), four initial values are required to fix a solution of Eq. (C3) and these are given with the selective property in Eq. (C19) for the $X_{i}(j)$ 's. We call the $X_{i}(j)$ basic or primitive Tetranacci polynomials.

A second proof that the $X_{i}(j)$ obey the recursion formula in Eq. (C3) follows directly from Eq. (C18). Choosing only one initial value different from zero, e.g., $\xi_{j}=\delta_{j 1}$ for $j=$ $-2, \ldots, 1$, results in

$$
\xi_{j}=X_{1}(j) .
$$

Similar choices reveal that $\xi_{j}$ can be equal to only one of the $X_{i}(j)$. Thus, the $X_{i}(j)$ must be Tetranacci polynomials.

The easier form of $x_{j, 0}$ in Eq. (C8) cannot be seen from here, since the $r_{ \pm i}$ does not reduce to the $R_{ \pm}$at $E=0$. The reason is that the recursion formulas for $E=0$ and $E \neq 0$ do not transform directly into each other. In the limiting case of $\mu=0$, we find from Eq. (C14) that

$$
\left.S_{1}\right|_{\mu=0}=-\left.S_{2}\right|_{\mu=0},
$$

yielding

$$
\left.r_{+1}\right|_{\mu=0}=-\left.r_{-2}\right|_{\mu=0} .
$$

The effect on $F_{1,2}$ in Eqs. (C20) and (C21) is

$$
\left.F_{1}(j)\right|_{\mu=0}=\left.(-1)^{j-1} F_{2}(j)\right|_{\mu=0},
$$


and we find further that

$$
\begin{aligned}
\left.X_{-2}(2 l+1)\right|_{\mu=0} & =0, \\
\left.X_{0}(2 l+1)\right|_{\mu=0} & =0, \\
\left.X_{-1}(2 l)\right|_{\mu=0} & =0, \\
\left.X_{1}(2 l)\right|_{\mu=0} & =0,
\end{aligned}
$$

for all values of $l$. Thus, the form of the recursion formula at $\mu=0$ in Eq. (C3) is respected and the Tetranacci polynomials $\xi_{j}$ reduce back to Fibonacci polynomials for $\mu=0$. This behavior of $\xi_{j}$ can be also understood in a different way. The definition of the Tetranacci polynomial $F_{1,2}$ is actually a Binet form of Fibonacci polynomials [60-62]. One can easily prove that $F_{1,2}$ obey

$$
F_{i}(j+2)=S_{i} F_{i}(j+1)-F_{i}(j),
$$

for all $j$, with $F_{1,2}(0)=0, F_{1,2}(1)=1$. Thus, the closed form of $\xi_{j}$ can be seen as a superposition of two distinct sequences of Fibonacci polynomials $F_{1,2}$.

However, one has to account for all four different fundamental solutions $r_{ \pm i}(i=1,2)$ in the case of Tetranacci polynomials. Similar to the denominator of a Binet form, which contains the difference of the two fundamental solutions of the corresponding Fibonacci sequence $\left[r_{+i}-r_{-i}\right.$ for $F_{i}(j)$ ], we find that $S_{1,2}$ adopt this role in the case of Tetranacci polynomials. Note that $S_{1}-S_{2}=r_{+1}+r_{-1}-r_{+2}-r_{-2}$.

\section{APPENDIX D: EIGENVECTORS AND DEGENERACIES IN THE SPECTRUM}

\section{The general eigenvector problem}

We briefly recapitulate here the eigenvector problem investigated in Ref. [39] and introduce the inversion symmetry before we turn to the degenerate energy eigenvalues. The Kitaev chain Hamiltonian can be expressed in the chiral basis [cf. Eq. (B2)] through

$$
\mathcal{H}_{c}=\left[\begin{array}{cc}
0_{N \times N} & h \\
h^{\dagger} & 0_{N \times N}
\end{array}\right],
$$

with $\hat{H}_{\mathrm{KC}}=\frac{1}{2} \hat{\Psi}_{c}^{\dagger} \mathcal{H}_{c} \hat{\Psi}_{c}$ and $h_{n, m}=-i \mu \delta_{n m}+a \delta_{n, m-1}-$ $b \delta_{n, m+1}$ for $n, m=1, \ldots, N, a=i(\Delta-t), b=i(\Delta+t)$. For an eigenvector $\vec{w}=\left(\vec{v}_{A}, \vec{v}_{B}\right)^{\mathrm{T}}$ of the Hamiltonian $\mathcal{H}_{c}$, the sublattice vectors $\vec{v}_{A}:=\left(\xi_{1} \ldots, \xi_{N}\right)^{\mathrm{T}}, \vec{v}_{B}:=\left(\sigma_{1} \ldots, \sigma_{N}\right)^{\mathrm{T}}$ have to obey

$$
\begin{aligned}
& h \vec{v}_{B}=E \vec{v}_{A}, \\
& h^{\dagger} \vec{v}_{A}=E \vec{v}_{B} .
\end{aligned}
$$

In particular, we consider here exclusively the case of $E \neq$ 0 , where one can choose all $\xi_{n}\left(\sigma_{n}\right)$ as real (pure imaginary) numbers. Solving for $\vec{v}_{A}$ grants

$$
h h^{\dagger} \vec{v}_{A}=E^{2} \vec{v}_{A},
$$

and $\vec{v}_{B}$ follows then from Eq. (D3). Importantly, Eq. (D4) directly implies that the entries of $\vec{v}_{A}$ obey the Tetranacci recursion formula:

$$
\begin{aligned}
\xi_{j+2}= & \frac{E^{2}+a^{2}+b^{2}-\mu^{2}}{a b} \xi_{j}-\xi_{j-2} \\
& +i \mu \frac{b-a}{a b}\left(\xi_{j-1}-\xi_{j+1}\right) .
\end{aligned}
$$

Extending the sequence of $\xi$ 's via Eq. (D5) beyond the range $j=1, \ldots, N$ allows the simplification of the boundary condition to

$$
\xi_{0}=\xi_{N+1}=b \xi_{1}-a \xi_{-1}=b \xi_{N+2}-a \xi_{N}=0,
$$

while $\vec{v}_{A}$ still contains only $\xi_{1}, \ldots, \xi_{N}$ and the boundary condition yields after some algebra the quantization rule [39] given by Eq. (6). The values of $\kappa_{1,2}$ and $E$ are thus fixed. A sequence obeying Eq. (D5) requires four initial values, for example, $\xi_{-2}, \xi_{-1}, \xi_{0}, \xi_{1}$, and one can derive the following closed formula:

$$
\xi_{j}=\sum_{i=-2}^{1} \xi_{i} X_{i}(j),
$$

where the functions $X_{i}(j)$ [cf. Eqs. (C22)-(C24) in Appendix C 2] depend on $E^{2}, t, \Delta, \mu$, and $N$; they inherit the selective property $X_{i}(j)=\delta_{i, j}$ for only $i, j=-2, \ldots, 1$. As we see from the boundary conditions in Eq. (D6), $\xi_{0}=0$ and thus is fixed, while $\xi_{-1}=b \xi_{1} / a$. In the case of no degeneracy, we have one degree of freedom and we can choose $\xi_{1}$ arbitrarily. Consequently, $\xi_{-2}$ is the last missing initial value and can be fixed via $\xi_{N+1}=0$, yielding

$$
\xi_{-2}=-\xi_{1} \frac{a X_{1}(N+1)+b X_{-1}(N+1)}{a X_{-2}(N+1)}
$$

in the absence of degeneracy and with $X_{-2}(N+1) \neq 0$. If $X_{-2}(N+1)=0$, we should use the fourth equality in Eq. (D6) instead. The eigenvector problem is now solved, since the constraint $b \xi_{N+2}-a \xi_{N}=0$ quantizes the wave vectors generating the eigenvalue $E$ and thus the values of the functions $X_{i}(j)$ are known.

\section{Inversion symmetry}

In our previous work, we used a rather lengthy method to determine the entries of $\vec{v}_{B}$ from the ones of $\vec{v}_{A}$. The inversion symmetry $I$ allows us to pursue a much simpler method as we explain in the following. The modified inversion symmetry of the Kitaev chain which we discussed in Sec. II, i.e., the invariance of $\hat{H}_{\mathrm{KC}}$ under the exchange $d_{j} \rightarrow i d_{N+1-j}, d_{j}^{\dagger} \rightarrow$ $-i d_{N+1-j}^{\dagger}$, involves an additional global phase of $i$ for the Nambu spinor. The consequences of the simple inversion symmetry of the original chain $\left(I: d_{j}^{(\dagger)} \rightarrow d_{N+1-j}^{(\dagger)}\right)$ can, however, be explored in a more elegant way, which does not need to introduce an additional phase. In the BdG basis, $I \hat{H}_{\mathrm{KC}} I^{-1}=$ $\left.\hat{H}_{\mathrm{KC}}\right|_{-\Delta}$. Written in the basis of $\mathcal{H}_{c}$, the representation of $I$ is

$$
I_{c}=\left[\begin{array}{ll}
I_{0} & \\
& I_{0}
\end{array}\right], \quad I_{0}=\left[\begin{array}{lll} 
& & . \\
1 & &
\end{array}\right],
$$

where $I_{0}$ represents the usual inversion operation, i.e., reversing the site order. The use of $I_{c}$ on the eigenvector problem yields

$$
\left(\left.h\right|_{-\Delta}\right) I_{0} \vec{v}_{B}=E I_{0} \vec{v}_{A}, \quad\left(\left.h^{\dagger}\right|_{-\Delta}\right) I_{0} \vec{v}_{A}=E I_{0} \vec{v}_{B} .
$$

Importantly, we have that $\left.h\right|_{-\Delta}=-h^{\dagger}$ and vice versa, transforming the equations for $I_{0} \vec{v}_{A}\left(I_{0} \vec{v}_{B}\right)$ in the ones of $\vec{v}_{B}\left(\vec{v}_{A}\right)$ at $-E$. Recalling that all $\vec{v}_{A}\left(\vec{v}_{B}\right)$ are real (pure imaginary) 
vectors, we can cancel this sign of $E$ by the now obvious relation between $\vec{v}_{A}$ and $\vec{v}_{B}$ :

$$
\begin{aligned}
& \vec{v}_{A}= \pm i I_{0} \vec{v}_{B}, \\
& \vec{v}_{B}=\mp i I_{0} \vec{v}_{A} .
\end{aligned}
$$

The sign of $E$ to which the \pm solution belongs, depends on the parameters. Thus, the entries of $\vec{v}_{B}$ now obey the simple relation $\sigma_{N+1-j}=\mp i \xi_{j}$ and a normalized eigenvector $\vec{w}$ is achieved by normalizing $\vec{v}_{A}$ and division by $\sqrt{2}$.

In the special case of $E=0$, the degenerate eigenstates are still related by inversion symmetry but the decoupling of $\vec{v}_{A}$ and $\vec{v}_{B}$ always allows us to set one of them to zero, whereby a relation between $\vec{v}_{A}$ and $\vec{v}_{B}$ of the same eigenvector can become invalid.

Once $\vec{v}_{A}$ is known, one can rewrite the solution in the basis of the fermionic operators $d_{j}^{(\dagger)}$. After the transformation the electron $d_{j}$ (hole $d_{j}^{\dagger}$ ) part of the quasiparticle state is $\vec{v}_{A}-i \vec{v}_{B}\left(\vec{v}_{A}+i \vec{v}_{B}\right)$. The different combination signals opposite behavior under inversion symmetry as captured in Fig. 3 . Further, after an application of the particle-hole symmetry to the eigenstates, the character of the electron and hole parts under inversion symmetry changes into the opposite, since the exchange $E \rightarrow-E$ means $\vec{v}_{B} \rightarrow-\vec{v}_{B}$ while keeping the same $v_{A}$.

\section{Degenerate energy levels}

The important starting point for the case of degeneracies is Fig. 2, where we see that for specific values of $t, \Delta$ and $\mu$ a crossing in the spectrum occurs, which naturally depends also on $N$. We exclude here from consideration the cases of $E=0, t^{2}=\Delta^{2}(a b=0)$, and $t \Delta=0$ because they are already known. Further, we consider $t, \Delta$ as fixed while $\mu$ can be varied to achieve a degeneracy.

Let us begin by inspecting the degree of the degeneracy and assume initially that we have $D \geqslant 2$ degenerate eigenvectors $\vec{v}^{(d)}=\left(\vec{v}_{A}^{(d)}, \vec{v}_{B}^{(d)}\right)^{\mathrm{T}}$ with $d=1, \ldots, D$ and all $\vec{v}^{(d)}$ have to obey the Eqs. (D2)-(D7). We continue with almost the same notation as above, where we change only $\xi_{j}\left(\sigma_{j}\right)$ into $\xi_{j}^{(d)}$ $\left(\sigma_{j}^{(d)}\right)$ for clarity. The eigenstates are still determined by the quantization rule in Eq. (6), and in the following we will obtain the required further constraint on Eq. (6) needed for the eigenstates to be degenerate.

The case of degenerate eigenvectors has to be treated carefully, since their superposition can break the connection between $\vec{v}_{A}^{(d)}$ and $\vec{v}_{B}^{(d)}$ via inversion symmetry. Nonetheless, once the value of the energy is known, all information of $\vec{v}^{(d)}$ is still contained in $\vec{v}_{A}^{(d)}$, since $\vec{v}_{B}^{(d)}$ follows from $h^{\dagger} \vec{v}_{A}^{(d)}=$ $E \vec{v}_{B}^{(d)}$. Furthermore, for given values of $t, \Delta$, and $\mu$, the functions $X_{i}(j)$ in Eq. (D7) differ only for states with different energies, therefore the $\vec{v}_{A}^{(d)}$ are defined only by distinct initial values $\xi_{-2}^{(d)}, \ldots, \xi_{1}^{(d)}$. Thus one can build and exploit special superpositions of those eigenstates, yielding

$$
\begin{aligned}
& \xi_{1}^{(1)}=1, \quad \xi_{-2}^{(1)}=0, \\
& \xi_{1}^{(2)}=0, \quad \xi_{-2}^{(2)}=1 .
\end{aligned}
$$

The boundary condition in Eq. (D6) demands $\xi^{(0)}=0, \xi_{-1}^{(d)}=$ $b \xi_{1}^{(d)} / a$ and thus fixes $\vec{v}_{A}^{(d)}$. Note that Eqs. (D11) and (D12) imply $D=2$, i.e., only twofold degeneracies are allowed, since beyond $\xi_{1}$ and $\xi_{-2}$ there are no further degrees of freedom to exploit. As we see next, Eq. (D8), which formerly coupled $\xi_{1}^{(d)}$ and $\xi_{-2}^{(d)}$, becomes indeed invalid for the new superpositions. Returning to the boundary condition in Eq. (D6), we get further constraints, namely,

$$
\begin{gathered}
X_{-2}(N+1)=0, \\
b X_{-2}(N+2)-a X_{-2}(N)=0, \\
a X_{1}(N+1)+b X_{-1}(N+1)=0, \\
b\left[a X_{1}(N+2)+b X_{-1}(N+2)\right] \\
-a\left[a X_{1}(N)+b X_{-1}(N)\right]=0,
\end{gathered}
$$

implying a division of zero by zero in Eq. (D8). Further, Eqs. (D13)-(D16) show that the boundary condition splits into two parts for $N+1$ and for $N+2, N$ which is the constraint on Eq. (6) for which we have been looking.

As we have discussed in Appendix C 2, the four functions $X_{i}(j)$ are constructed with the help of two special functions $F_{1,2}$, see Eqs. (C22)-(C24), which are are nothing else than standing waves,

$$
F_{1,2}(j)=\frac{\sin \left(\kappa_{1,2} j\right)}{\sin \left(\kappa_{1,2}\right)},
$$

at site $j$, constructed from the plane waves $r_{+1,+2}=e^{i \kappa_{1,2}}$ as follows from Eqs. (C15), (C20), and (C21).

Now we can solve for $\kappa_{1,2}$. We get first from Eq. (D13) two constraints: $S_{1}-S_{2} \neq 0$, i.e., $\kappa_{1} \neq \pm \kappa_{2}$, and $F_{1}(N+1)=$ $F_{2}(N+1)$. Second, these two restrictions used on Eq. (D15) together with exploiting the properties of $F_{1,2}$ [for example, Eq. (C26)], give us a familiar expression, namely,

$$
a X_{-2}(N+2)-b X_{-2}(N)=0,
$$

which is almost Eq. (D14). Thus, $X_{-2}(N+2)=X_{-2}(N)=0$ holds or, equivalently, $F_{1}(N+2)=F_{2}(N+2)$ and $F_{1}(N)=$ $F_{2}(N)$. This imposes a further constraint on $\kappa_{1,2}$ to obey $F_{1,2}(N+1)=0$. Thus $\kappa_{1,2}=n \pi /(N+1), n=1, \ldots, N$.

The combinations of different values of $\kappa_{1,2}$ yield both the positions of strict and of avoided crossings in the $(\mu, E)$ plane. The values of $\mu$ follow from Eq. (5) after converting the values of $\kappa_{1,2}$ into $\kappa_{\Sigma, \Delta}$. The energy $E$, in turn, can be obtained from the dispersion relation in Eq. (2). Whether these $(\mu, E)$ pairs define strict or avoided crossings is determined by the general quantization rule in Eq. (6), considering the following facts: (i) With $\kappa_{1,2}=n_{1,2} \pi /(N+1)$, the values for $\kappa_{\Sigma, \Delta}$ are either both half integer or both integer multiples of $\pi /(N+1)$. (ii) The entire derivation for $\kappa_{1,2}$ is invariant under the exchange $\kappa_{1} \rightarrow \pm \kappa_{2}$ and $\kappa_{1} \rightarrow-\kappa_{1}$, hence, without loss of generality we can demand $\kappa_{1}>\kappa_{2}$. (iii) By virtue of Eq. (5), $\kappa_{1}+\kappa_{2} \neq \pi$, except for $N$ odd and $\mu=0$. In the end, we find that only $\kappa_{\Sigma, \Delta}$ which are integer multiples of $\pi /(N+1)$ satisfy the quantization rule Eq. (6) for an arbitrary value of $\Delta$. Thus, the selection rule for strict crossings can be expressed in terms of $\kappa_{\Sigma, \Delta}$, demanding that $\kappa_{\Sigma}>\kappa_{\Delta}$, and 
resulting in the requirement of $\kappa_{\Sigma, \Delta}$ being integer multiples of $\pi /(N+1)$ as stated in Eqs. (7) and (8). The half-integer multiples satisfy Eq. (6) only if $\Delta=0$, hence in a superconducting chain they always define avoided crossings.

\section{APPENDIX E: DERIVATION OF THE CURRENT FORMULA}

The electronic current (for fixed spin) in the left lead is

$$
I_{L}(t)=-e\left\langle\dot{N}_{L}\right\rangle
$$

where $e$ is the elementary charge and $N_{L}=\sum_{k} c_{k L}^{\dagger} c_{k L}$. The specific choice of the tunneling Hamiltonian $H_{\mathrm{T}}$ in Eqs. (10) leads to the explicit expression

$$
I_{L}(t)=-\frac{i e}{\hbar} \sum_{k}\left(t_{L}\left\langle d_{1}^{\dagger}(t) c_{k L}(t)\right\rangle-t_{L}^{*}\left\langle c_{k L}^{\dagger}(t) d_{1}(t)\right\rangle\right),
$$

where the superconductivity is contained inside the time evolution of the creation and annihilation operators. Further, we shall use a $2 \times 2$ matrix notation for the fermionic Green's functions whose entries are defined via $D_{j}:=\left(d_{j}, d_{j}^{\dagger}\right)^{\mathrm{T}}$ as

$$
\begin{gathered}
\left(\mathbf{G}_{i j}^{>}\left(t, t^{\prime}\right)\right)_{n m}:=-\frac{i}{\hbar}\left\langle\left(D_{i}(t)\right)_{n}\left(D_{j}\left(t^{\prime}\right)\right)_{m}^{\dagger}\right\rangle, \\
\left(\mathbf{G}_{i j}^{<}\left(t, t^{\prime}\right)\right)_{n m}:=\frac{i}{\hbar}\left\langle\left(D_{j}\left(t^{\prime}\right)\right)_{m}^{\dagger}\left(D_{i}(t)\right)_{n}\right\rangle, \\
\left(\mathbf{G}_{i j}^{r}\left(t, t^{\prime}\right)\right)_{n m}:=-\frac{i}{\hbar} \theta\left(t-t^{\prime}\right)\left\langle\left\{\left(D_{i}(t)\right)_{n},\left(D_{j}\left(t^{\prime}\right)\right)_{m}^{\dagger}\right\}\right\rangle, \\
\left(\mathbf{G}_{i j}^{a}\left(t, t^{\prime}\right)\right)_{n m}:=\frac{i}{\hbar} \theta\left(t^{\prime}-t\right)\left\langle\left\{\left(D_{i}(t)\right)_{n},\left(D_{j}\left(t^{\prime}\right)\right)_{m}^{\dagger}\right\}\right\rangle,
\end{gathered}
$$

where $\{\cdot, \cdot\}$ denotes the anticommutator and $n, m=1,2$. All kinds of Green's functions, such as $\mathbf{G}_{k \alpha}^{s}\left(t, t^{\prime}\right), \mathbf{G}_{j k \alpha}^{s}\left(t, t^{\prime}\right)$ for $s=\langle, r, a$,$\rangle , are defined analogously with C_{k \alpha}:=\left(c_{k \alpha}, c_{k \alpha}^{\dagger}\right)^{\mathrm{T}}$ instead of $D_{j}$. Please keep in mind that the NEGF formalism uses a large variety of inter-related Green's functions.

In the following, we will denote all $2 \times 2$ matrices with a bold font to keep them distinct from the $2 N \times 2 N$ matrices used everywhere else.

A convenient expression for the current in terms of those $2 \times 2$ matrices is

$$
I_{L}(t)=-e \sum_{k} \operatorname{Re}\left\{\operatorname{Tr}\left[\left(\begin{array}{cc}
t_{L} & 0 \\
0 & t_{L}^{*}
\end{array}\right) \mathbf{G}_{k L 1}^{<}(t, t)\right]\right\} .
$$

Starting from the equation of motion for the relevant Green's functions, and using standard relations between them together with the Langreth rules [48-50,54,63], we find the steadystate current

$$
\begin{aligned}
I_{L}=- & e \int_{\mathbb{R}} \frac{d \omega}{2 \pi} \operatorname{Tr}\left\{\tau _ { z } \left[\boldsymbol{\Sigma}_{L}^{r}(\omega) \mathbf{G}_{11}^{<}(\omega)\right.\right. \\
& \left.\left.+\boldsymbol{\Sigma}_{L}^{<}(\omega) \mathbf{G}_{11}^{a}(\omega)\right]\right\},
\end{aligned}
$$

with

$$
\begin{aligned}
\Sigma_{\alpha}^{r}(\omega)= & \lim _{\eta \rightarrow 0} \sum_{k}\left|t_{\alpha}(k)\right|^{2}\left[\begin{array}{cc}
\frac{1}{\hbar \omega-\epsilon_{k \alpha}+i \eta} & 0 \\
0 & \frac{1}{\hbar \omega+\epsilon_{k \alpha}+i \eta}
\end{array}\right], \\
\boldsymbol{\Sigma}_{\alpha}^{<}(\omega)=2 & \pi i \sum_{k}\left|t_{\alpha}(k)\right|^{2}\left[\begin{array}{cc}
\delta\left(\hbar \omega-\epsilon_{k \alpha}\right) & 0 \\
0 & \delta\left(\hbar \omega+\epsilon_{k \alpha}\right)
\end{array}\right] \\
& \times\left[\begin{array}{cc}
f\left(\hbar \omega-e V_{\alpha}\right) & 0 \\
0 & f\left(\hbar \omega+e V_{\alpha}\right)
\end{array}\right] .
\end{aligned}
$$

The lesser Green's function matrices $\mathbf{G}_{11}^{<, a}$ involve only the first site of the Kitaev chain and carry information about the coupling of this site with both the rest of the chain and the leads. To obtain them, it is convenient to work in the site-ordered particle-hole basis [cf. Eq. (B3)], where the $2 \times 2$ matrices introduced above become the building blocks of $\tilde{G}^{s}$ $(s=\langle, r, a\rangle$,$) :$

$$
\tilde{G}^{s}=\left[\begin{array}{ccc}
\mathbf{G}_{11}^{s} & \ldots & \mathbf{G}_{1 N}^{s} \\
\vdots & & \vdots \\
\mathbf{G}_{N 1}^{s} & \ldots & \mathbf{G}_{N N}^{s}
\end{array}\right] .
$$

We find that $\tilde{G}^{r}$ obeys

$$
\left[(\hbar \omega+i \eta) \mathbb{1}_{2 N}-\tilde{\mathcal{H}}-\tilde{\Sigma}_{L}^{r}-\tilde{\Sigma}_{R}^{r}\right] \tilde{G}^{r}=\mathbb{1}_{2 N},
$$

with the self-energy matrices $\tilde{\Sigma}_{L, R}^{s}(s=\langle, r, a\rangle$,$) given by$

$$
\begin{aligned}
\tilde{\Sigma}_{L}^{s}= & {\left[\begin{array}{cccc}
\boldsymbol{\Sigma}_{L}^{s} & \mathbf{0} & \ldots & \mathbf{0} \\
\mathbf{0} & \mathbf{0} & \ldots & \mathbf{0} \\
\vdots & \vdots & \ddots & \vdots \\
\mathbf{0} & \mathbf{0} & \ldots & \mathbf{0}
\end{array}\right]_{2 N \times 2 N}, } \\
\tilde{\Sigma}_{R}^{s}= & {\left[\begin{array}{cccc}
\mathbf{0} & \ldots & \mathbf{0} & \mathbf{0} \\
\vdots & \ddots & \vdots & \vdots \\
\mathbf{0} & \ldots & \mathbf{0} & \mathbf{0} \\
\mathbf{0} & \ldots & \mathbf{0} & \boldsymbol{\Sigma}_{R}^{s}
\end{array}\right]_{2 N \times 2 N}, }
\end{aligned}
$$

where $\mathbf{0}$ is the $2 \times 2$ matrix filled with zeros. The Hamiltonian $\tilde{\mathcal{H}}$ reads

$$
\tilde{\mathcal{H}}=\left[\begin{array}{ccccc}
-\mu \tau_{z} & \boldsymbol{\alpha} & & & \\
\boldsymbol{\alpha}^{\dagger} & -\mu \tau_{z} & \boldsymbol{\alpha} & & \\
& \ddots & \ddots & \ddots & \\
& & \boldsymbol{\alpha}^{\dagger} & -\mu \tau_{z} & \boldsymbol{\alpha} \\
& & & \boldsymbol{\alpha}^{\dagger} & -\mu \tau_{z}
\end{array}\right]_{2 N \times 2 N}
$$

where we kept the Pauli matrix $\tau_{z}$ in regular font, and the matrix $\alpha$

$$
\boldsymbol{\alpha}=\left[\begin{array}{cc}
-t & -\Delta \\
\Delta & t
\end{array}\right]
$$

accounts for nearest-neighbor terms. Further, $\tilde{G}^{<}$obeys

$$
\tilde{G}^{<}=\tilde{G}^{r}\left(\tilde{\Sigma}_{L}^{<}+\tilde{\Sigma}_{R}^{<}\right) \tilde{G}^{a},
$$

with $\tilde{G}^{a}=\left(\tilde{G}^{r}\right)^{\dagger}$ so all ingredients of Eq. (E8) are in principle known. The trace and the sparsity of the self-energies $\tilde{\Sigma}_{L, R}^{s}$ allow us to express the current

$$
\begin{aligned}
I_{L}= & -e \int_{\mathbb{R}} \frac{d \omega}{2 \pi} \operatorname{Tr}\left\{\mathbb { 1 } _ { N } \otimes \tau _ { z } \left[\tilde{\Sigma}_{L}^{r}(\omega) \tilde{G}^{<}(\omega)\right.\right. \\
& \left.\left.+\tilde{\Sigma}_{L}^{<}(\omega) \tilde{G}^{a}(\omega)\right]\right\}
\end{aligned}
$$


in terms of these $2 N \times 2 N$ matrices. We define $\tilde{\Gamma}_{\alpha}:=$ $-2 \operatorname{Im}\left(\tilde{\Sigma}_{\alpha}^{r}\right)=i\left(\tilde{\Sigma}_{\alpha}^{r}-\tilde{\Sigma}_{\alpha}^{a}\right)$ and with

$$
\tilde{F}_{\alpha}=\mathbb{1}_{N} \otimes\left[\begin{array}{cc}
f\left(\hbar \omega-e V_{\alpha}\right) & 0 \\
0 & f\left(\hbar \omega+e V_{\alpha}\right)
\end{array}\right],
$$

it follows that $\tilde{\Sigma}_{\alpha}^{<}=\tilde{\Gamma}_{\alpha} \tilde{F}_{\alpha}$. Since the current is a real quantity, i.e., $2 I_{L}=I_{L}+I_{L}^{\dagger}$, we find the appealing form [52]

$$
I_{L}=i \frac{e}{2} \int_{\mathbb{R}} \frac{d \omega}{2 \pi} \operatorname{Tr}\left\{\left(\mathbb{1}_{N} \otimes \tau_{z}\right) \tilde{\Gamma}_{L}\left[\tilde{G}^{<}+\tilde{F}_{L}\left(\tilde{G}^{r}-\tilde{G}^{a}\right)\right]\right\} .
$$

Expressing all quantities in the default basis Eq. (B1) yields directly Eq. (12). The corresponding expressions of the self-energies and Green's functions are explicitly given in Appendix F.

Finding the analytical form of the conductance demands first a simplification toward Eq. (15), which mostly consists of taking the trace and using the sparsity of the self-energies. This procedure is performed at best by using Eq. (E8) and a basis transformation Eq. (B4) at the end. We find from Eq. (E17) that

$$
\mathbf{G}_{11}^{<}=\mathbf{G}_{11}^{r} \mathbf{\Sigma}_{L}^{<} \mathbf{G}_{11}^{a}+\mathbf{G}_{1 N}^{r} \mathbf{\Sigma}_{R}^{<} \mathbf{G}_{N 1}^{a}
$$

and Eq. (E12) yields first $\tilde{G}^{r}-\tilde{G}^{a}=-i \tilde{G}^{r}\left(\tilde{\Gamma}_{L}+\tilde{\Gamma}_{R}\right) \tilde{G}^{a}$ and thus

$$
\mathbf{G}_{11}^{r}-\mathbf{G}_{11}^{a}=-i\left[\mathbf{G}_{11}^{r} \boldsymbol{\Gamma}_{L} \mathbf{G}_{11}^{a}+\mathbf{G}_{1 N}^{r} \boldsymbol{\Gamma}_{R} \mathbf{G}_{N 1}^{a}\right] .
$$

With $2 I_{L}=I_{L}+I_{L}^{\dagger}$, it follows from Eq. (E8) that

$$
\begin{aligned}
I_{L}= & i \frac{e}{2} \int_{\mathbb{R}} \frac{d \omega}{2 \pi} \operatorname{Tr}\left\{\tau _ { z } \left[\boldsymbol{\Gamma}_{L} \mathbf{G}_{11}^{r} \boldsymbol{\Sigma}_{L}^{<} \mathbf{G}_{11}^{a}\right.\right. \\
& -\boldsymbol{\Sigma}_{L}^{<} \mathbf{G}_{11}^{r} \boldsymbol{\Gamma}_{L} \mathbf{G}_{11}^{a} \\
& +\boldsymbol{\Gamma}_{L} \mathbf{G}_{1 N}^{r} \boldsymbol{\Sigma}_{R}^{<} \mathbf{G}_{N 1}^{a} \\
& \left.\left.-\boldsymbol{\Sigma}_{L}^{<} \mathbf{G}_{1 N}^{r} \boldsymbol{\Gamma}_{R} \mathbf{G}_{N 1}^{a}\right]\right\},
\end{aligned}
$$

where the $2 \times 2$ broadening matrices read

$$
\Gamma_{\alpha}=\left[\begin{array}{cc}
\Gamma_{\alpha}^{-} & 0 \\
0 & \Gamma_{\alpha}^{+}
\end{array}\right]
$$

with the abbreviations $\Gamma_{\alpha}^{ \pm}=2 \pi \sum_{k}\left|t_{\alpha}(k)\right|^{2} \delta\left(\hbar \omega \pm \epsilon_{k \alpha}\right)$. To shorten the expression of the trace, we define

$$
f_{\alpha}^{ \pm}:=f\left(\hbar \omega \pm e V_{\alpha}\right),
$$

and after a bit of algebra one finds

$$
\begin{aligned}
i \operatorname{Tr}\left\{\tau_{z}\left[\boldsymbol{\Gamma}_{L} \mathbf{G}_{11}^{r} \boldsymbol{\Sigma}_{L}^{<} \mathbf{G}_{11}^{a}-\boldsymbol{\Sigma}_{L}^{<} \mathbf{G}_{11}^{r} \boldsymbol{\Gamma}_{L} \mathbf{G}_{11}^{a}\right]\right\} \\
=\Gamma_{L}^{-} \Gamma_{L}^{+}\left(\left|\tilde{G}_{1,2}^{r}(\omega)\right|^{2}+\left|\tilde{G}_{2,1}^{r}(\omega)\right|^{2}\right)\left[f_{L}^{-}-f_{L}^{+}\right] \\
i \operatorname{Tr}\left\{\tau_{z}\left[\boldsymbol{\Gamma}_{L} \mathbf{G}_{1 N}^{r} \boldsymbol{\Sigma}_{R}^{<} \mathbf{G}_{N 1}^{a}-\boldsymbol{\Sigma}_{L}^{<} \mathbf{G}_{1 N}^{r} \boldsymbol{\Gamma}_{R} \mathbf{G}_{N 1}^{a}\right]\right\} \\
=\Gamma_{L}^{-} \Gamma_{R}^{-}\left|\tilde{G}_{1,2 N-1}^{r}(\omega)\right|^{2}\left[f_{L}^{-}-f_{R}^{-}\right] \\
\quad+\Gamma_{L}^{+} \Gamma_{R}^{+}\left|\tilde{G}_{2,2 N}^{r}(\omega)\right|^{2}\left[f_{R}^{+}-f_{L}^{+}\right] \\
\quad+\Gamma_{L}^{-} \Gamma_{R}^{+}\left|\tilde{G}_{1,2 N}^{r}(\omega)\right|^{2}\left[f_{L}^{-}-f_{R}^{+}\right] \\
\quad+\Gamma_{L}^{+} \Gamma_{R}^{-}\left|\tilde{G}_{2,2 N-1}^{r}(\omega)\right|^{2}\left[f_{R}^{-}-f_{L}^{+}\right] .
\end{aligned}
$$

In contrast to Eq. (15), where only electronic contributions are used, in Eqs. (E26) and (E27) we have six terms for both electronic and hole degrees of freedom, and a factor of $1 / 2$ in front of Eq. (E23) to avoid overcounting. The following steps will further reduce the number of terms.

Throughout our approach, we considered $t$ and $\Delta$ as real quantities. Hence, $\tilde{\mathcal{H}}$ is a symmetric matrix. Since $\tilde{\Sigma}_{\alpha}^{r}$ are symmetric too, we have that $\tilde{G}_{i, j}^{r}=\tilde{G}_{j, i}^{r}$. This yields in Eq. (E26) a factor of 2 .

Further, the particle-hole symmetry gives

$$
\left(\mathbb{1}_{N} \otimes \sigma_{x}\right)\left[\tilde{G}^{r}(-\omega)\right]^{*}\left(\mathbb{1}_{N} \otimes \sigma_{x}\right)=-\tilde{G}^{r}(\omega),
$$

where $*$ denotes the complex conjugation. The use of Eq. (E28) on $\tilde{G}^{r}(\omega)$ and observing its particular action on the entries of the $2 \times 2$ block $\mathbf{G}_{1 N}^{r}$ yields

$$
\begin{aligned}
& \tilde{G}_{2,2 N}^{r}(\omega)=-\left[\tilde{G}_{1,2 N-1}^{r}(-\omega)\right]^{*}, \\
& \tilde{G}_{1,2 N}^{r}(\omega)=-\left[\tilde{G}_{2,2 N-1}^{r}(-\omega)\right]^{*} .
\end{aligned}
$$

Since $\Gamma_{\alpha}^{ \pm}(\omega)=\Gamma_{\alpha}^{\mp}(-\omega)$ holds, one has simply to split the integration in Eq. (E23) into two parts. After a substitution of $\omega \rightarrow-\omega$ and the use of the relations in Eqs. (E29) and (E30), we find that

$$
\begin{aligned}
I_{L}= & e \int_{\mathbb{R}} \frac{d \omega}{2 \pi}\left\{\Gamma_{L}^{-}(\omega) \Gamma_{L}^{+}(\omega)\left|\tilde{G}_{1,2}^{r}(\omega)\right|^{2}\left[f_{L}^{-}-f_{L}^{+}\right]\right. \\
& +\Gamma_{L}^{-}(\omega) \Gamma_{R}^{-}(\omega)\left|\tilde{G}_{1,2 N-1}^{r}(\omega)\right|^{2}\left[f_{L}^{-}-f_{R}^{-}\right] \\
& \left.+\Gamma_{L}^{-}(\omega) \Gamma_{R}^{+}(\omega)\left|\tilde{G}_{1,2 N}^{r}(\omega)\right|^{2}\left[f_{L}^{-}-f_{R}^{+}\right]\right\},
\end{aligned}
$$

which is already very close to Eq. (15); we need now a basis transformation given by Eq. (B4). The necessary entries of $\tilde{G}^{r}$ transform as

$$
\begin{aligned}
\tilde{G}_{1,2}^{r} & =G_{1, N+1}^{r}, \\
\tilde{G}_{1,2 N-1}^{r} & =G_{1, N}^{r}, \\
\tilde{G}_{1,2 N}^{r} & =G_{1,2 N}^{r},
\end{aligned}
$$

and inserting this in Eq. (E31) with the substitution $E=\hbar \omega$ leads almost directly to Eq. (15), though the bias still remains to be set.

The use of the mean-field technique breaks the conservation of the number of particles, if fixed values of $\Delta$ are used and thus $I_{L} \neq-I_{R}$. For correctness, one has to the use the self-consistently calculated profile of $\Delta$, since that correctly replaces two operators with their mean values and the number of particles is (implicitly) conserved. On the other side, one obviously prefers to avoid the self-consistency cycle. After we obtain $I_{R}$, we find that $I_{L}=-I_{R}$ holds for $\Gamma_{L}=\Gamma_{R}$ and symmetrically applied bias $\left(\eta=1 / 2\right.$, i.e., $\left.V_{L}=V / 2, V_{R}=-V / 2\right)$, without demanding the self-consistently calculated $\Delta[25,53]$. This trick sets the internal supercurrent to zero and allows the use of fixed values of $\Delta$. As a second effect, the crossed Andreev term $G_{1,2 N}^{r}$ does not contribute to the current, since the difference of the Fermi functions $f_{L}^{-}-f_{R}^{+}$is always zero for $\eta=1 / 2$ if both leads are at the same temperature. 


\section{APPENDIX F: MATRIX EXPRESSIONS IN THE (STANDARD) BOGOLIUBOV-DE GENNES BASIS}

The use of the default basis $\hat{\Psi}=$ $\left(d_{1}, \ldots, d_{N}, d_{1}^{\dagger}, \ldots, d_{N}^{\dagger}\right)^{\mathrm{T}}$ gives an intuitive understanding of the current formula, since the entries of the Hamiltonian, the self-energies, and the Green's functions are ordered first in the particle/hole subspace and second in the real space position. For example, $G_{1, N}^{r}$ describes the transport of an electron from site $j=1$ to site $j=N$, where it leaves the Kitaev chain as an electron to the right lead. We present here the matrices used in Eq. (12). The BdG Hamiltonian $\mathcal{H}$ reads

$$
\mathcal{H}=\left[\begin{array}{cc}
C & S \\
S^{\dagger} & -C
\end{array}\right]_{2 N \times 2 N},
$$

with $\hat{H}_{\mathrm{KC}}=\frac{1}{2} \hat{\Psi}^{\dagger} \mathcal{H} \hat{\Psi}, \hat{H}_{\mathrm{KC}}$ being given by Eq. (1). The matrices $C$ and $S$ are

$$
C=\left[\begin{array}{ccccccc}
-\mu & -t & & & & & \\
-t & -\mu & -t & & & & \\
& -t & -\mu & -t & & & \\
& & \ddots & \ddots & \ddots & & \\
& & & -t & -\mu & -t & \\
& & & & -t & -\mu & -t \\
& & & & & -t & -\mu
\end{array}\right]_{N \times N}
$$

$$
S=\left[\begin{array}{ccccccc}
0 & \Delta & & & & & \\
-\Delta & 0 & \Delta & & & & \\
& -\Delta & 0 & \Delta & & & \\
& & \ddots & \ddots & \ddots & & \\
& & & -\Delta & 0 & \Delta & \\
& & & & -\Delta & 0 & \Delta \\
& & & & & -\Delta & 0
\end{array}\right]_{N \times N}
$$

Due to the choice of the tunneling Hamiltonian $H_{L}$ in Eqs. (10), the self-energies $\Sigma_{L}^{r}$ and $\Sigma_{R}^{r}$ are sparse matrices $(i, j=1, \ldots, 2 N)$

$$
\begin{gathered}
\left(\Sigma_{L}^{r}\right)_{i, j}=\delta_{1 i} \delta_{1 j} \Omega_{L-}+\delta_{N+1, i} \delta_{N+1, j} \Omega_{L+}, \\
\left(\Sigma_{R}^{r}\right)_{i, j}=\delta_{N i} \delta_{N j} \Omega_{R-}+\delta_{i, 2 N} \delta_{2 N, j} \Omega_{R+}
\end{gathered}
$$

acting only on the first and last site. We used here the abbreviations

$$
\Omega_{\alpha \pm}=\lim _{\eta \rightarrow 0} \sum_{k} \frac{\left|t_{\alpha}(k)\right|^{2}}{E+i \eta \pm \epsilon_{k \alpha}}, \quad \alpha=L, R,
$$

where the index $-(+)$ accounts for particles (holes). In general, the finite lifetime introduced by the self-energies is given by the imaginary part $\operatorname{Im}\left(\Omega_{\alpha \pm}\right)=-\pi \sum_{k}\left|t_{\alpha}(k)\right|^{2} \delta(E \pm$ $\left.\epsilon_{k \alpha}\right)=:-\gamma_{\alpha}^{ \pm}$. In the special case of the wide band limit, the functions $\Omega_{\alpha \pm}$ don't depend on $E$ and become $\Omega_{\alpha \pm}=-i \gamma_{\alpha}$ from the main text.

Returning to the general case, the matrices $\Gamma_{\alpha}$ follow from

$$
\Gamma_{\alpha}(E)=-2 \operatorname{Im}\left(\Sigma_{\alpha}^{r}\right), \quad \alpha=L, R .
$$

The retarded Green's function $G^{r}$ is given by

$$
G^{r}=\left[E \mathbb{1}_{2 N}-\mathcal{H}_{\mathrm{BdG}}-\Sigma_{L}^{r}-\Sigma_{R}^{r}\right]^{-1},
$$

and the advanced Green's function obeys $G^{a}(E)=\left[G^{r}(E)\right]^{\dagger}$. The Fermi Dirac distribution $f(E)$ is contained in the matrix $F_{\alpha}$ such that

$$
F_{\alpha}=\left[\begin{array}{ll}
\mathbb{1}_{N} f\left(E-e V_{\alpha}\right) & \\
& \mathbb{1}_{N} f\left(E+e V_{\alpha}\right)
\end{array}\right],
$$

where $V_{\alpha}$ denotes the shift of the chemical potential at contact $\alpha=L, R$. Finally, the lesser Green's function $G^{<}(E)$ reads

$$
G^{<}(E)=i G^{r}\left[\sum_{\alpha=L, R} F_{\alpha} \Gamma_{\alpha}(E)\right] G^{a} .
$$

\section{APPENDIX G: THE EXACT FORM OF THE GREEN'S FUNCTIONS $G_{1, N+1}^{r}, G_{1, N}^{r}, G_{1,2 N}^{r}$}

The entries of the retarded Green's function $G_{1, N}^{r} G_{1, N+1}^{r}$ and $G_{1,2 N}^{r}$ in the default basis can be obtained analytically. The calculations are most conveniently performed in the siteordered Majorana basis defined in Eq. (B5), since the Kitaev Hamiltonian and the self-energies are reshaped into a block tridiagonal matrix, see Eq. (G1) below. Keeping in mind that $G^{s}=\mathrm{T}^{\dagger} G_{M}^{s} \mathrm{~T}$, after a bit of algebra one finds that

$$
\begin{aligned}
G_{1, N+1}^{r}= & \frac{1}{2}\left\{\left(G_{\mathrm{M}}^{r}\right)_{11}-\left(G_{\mathrm{M}}^{r}\right)_{22}+i\left[\left(G_{\mathrm{M}}^{r}\right)_{12}+\left(G_{\mathrm{M}}^{r}\right)_{21}\right]\right\} \\
G_{1, N}^{r}= & \frac{1}{2}\left\{\left(G_{\mathrm{M}}^{r}\right)_{1,2 N-1}+\left(G_{\mathrm{M}}^{r}\right)_{2,2 N}\right. \\
& \left.+i\left[\left(G_{\mathrm{M}}^{r}\right)_{2,2 N-1}-\left(G_{\mathrm{M}}^{r}\right)_{1,2 N}\right]\right\} . \\
G_{1,2 N}^{r}= & \frac{1}{2}\left\{\left(G_{\mathrm{M}}^{r}\right)_{1,2 N-1}-\left(G_{\mathrm{M}}^{r}\right)_{2,2 N}\right. \\
& \left.+i\left[\left(G_{\mathrm{M}}^{r}\right)_{2,2 N-1}+\left(G_{\mathrm{M}}^{r}\right)_{1,2 N}\right]\right\} .
\end{aligned}
$$

Although several other entries of the inverted matrix are required to obtain the entries $G_{1, N+1}^{r}, G_{1, N}^{r}$, and $G_{1,2 N}^{r}$ after the transformation, the inversion can be performed analytically. As it turns out, see Eq. (G2) below, the problem involves a nonlinear combination of polynomials and the basis transformation allows the decomposition.

In the case of $N \neq 1$, the retarded Green's function $G_{\mathrm{M}}^{r}$ is the inverse of

$$
\begin{aligned}
\mathcal{M}: & =E \mathbb{1}_{2 N}-\mathcal{H}_{\mathrm{M}}-\Sigma_{L, \mathrm{M}}^{r}-\Sigma_{R, \mathrm{M}}^{r} \\
& =\left[\begin{array}{cccccc}
\mathbf{A}_{L} & \mathbf{B} & & & & \\
\mathbf{C} & \mathbf{A}_{2} & \mathbf{B} & & & \\
& \mathbf{C} & \mathbf{A}_{3} & \mathbf{B} & & \\
& & \ddots & \ddots & \ddots & \\
& & & \mathbf{C} & \mathbf{A}_{N-1} & \mathbf{B} \\
& & & & \mathbf{C} & \mathbf{A}_{R}
\end{array}\right],
\end{aligned}
$$

with

$$
\begin{aligned}
\mathbf{A}_{j} & =\left[\begin{array}{cc}
E & i \mu \\
-i \mu & E
\end{array}\right], \quad \mathbf{C}^{\dagger}=\mathbf{B}=\left[\begin{array}{cc}
0 & -a \\
-b & 0
\end{array}\right], \\
\mathbf{A}_{\alpha} & =\mathbf{A}_{2}+\left[\begin{array}{cc}
\sigma_{\alpha, p} & i \sigma_{\alpha, m} \\
-i \sigma_{\alpha, m} & \sigma_{\alpha, p}
\end{array}\right],
\end{aligned}
$$

and $j=2, \ldots, N-1, a=i(\Delta-t), b=i(t+\Delta), \sigma_{\alpha, p}=$ $-\left(\Omega_{\alpha+}+\Omega_{\alpha-}\right) / 2, \sigma_{\alpha, m}=\left(\Omega_{\alpha+}-\Omega_{\alpha-}\right) / 2$. In the case of $N=1, G_{\mathrm{M}}^{r}$ is the inverse of

$$
\mathbf{A}_{2}+\sum_{\alpha=L, R}\left[\begin{array}{cc}
\sigma_{\alpha, p} & i \sigma_{\alpha, m} \\
-i \sigma_{\alpha, m} & \sigma_{\alpha, p}
\end{array}\right]
$$


The final results for $G_{1, N+1}^{r}, G_{1, N}^{r}$, and $G_{1,2 N}^{r}$ unite the cases $N=1, N \neq 1$ and so we drop this distinction. The required entries of $G_{\mathrm{M}}^{r}$ are obtained via the adjoint matrix technique, where one needs to calculate only determinants. The matrix form in Eq. (G1) allows us to use the method explained in Ref. [64], which entails the inversion of the B-type matrices. The calculation of $\operatorname{det}(\mathcal{M})$ is straightforward, but notice that the seemingly unimportant structure of the $\mathbf{B}$ matrices is the key here. The matrices $\mathbf{B}, \mathbf{B}^{-1}$ are off-diagonal, which yields simpler coefficients in the recursion formula and is the reason to use a basis of Majorana operators. A site-ordered fermionic basis [cf. Eq. (B3)] replaces B with $-\boldsymbol{\alpha}$ from Eq. (E16) and the calculation cannot be performed that easily.

Nevertheless, obtaining the entries of the adjoint matrix themselves requires even further tricks, which we cannot cover here. To give only one example, the minors of $\mathcal{M}$ which we have to calculate for the entries of $G^{r}$ are not of the same block tridiagonal form as $\mathcal{M}$ itself, one column and one row is missing. One has thus to extend the minors of $\mathcal{M}$ to $2 N \times 2 N$ without changing the value of the determinant, while at the same time restoring the same block tridiagonal shape. For the Andreev contributions, one has simply to add a row and a column, which contain only zeros except one single 1 at position $(1,1)$ of this new matrix. Laplace's expansion shows that the value of the determinant is unchanged, but the newly formed first upper/lower off-diagonal block is not invertible. To cure this, one has to consider an entire sequence of matrices, which converge back to the former, etc. Furthermore, once this calculation is accomplished, still a different procedure has to be adopted to calculate the direct and crossed Andreev terms.

We shall therefore simply give below the closed formulas for the relevant Green's functions and justify their form a posteriori. For example, if one calculates first $\operatorname{det}\left(E \mathbb{1}_{2 N}-\mathcal{H}_{\mathrm{M}}\right)$, which is essentially the characteristic polynomial and straightforward ${ }^{2}$ to derive with Ref. [64], one finds that

$$
\operatorname{det}\left(E \mathbb{1}_{2 N}-\mathcal{H}_{\mathrm{M}}\right)=(-a b)^{N}\left(x_{N} \mathcal{Y}_{N}-y_{N} \chi_{N}\right),
$$

where the functions $x_{N}, y_{N}, y_{N}, \chi_{N}$ are Tetranacci polynomials of order $N$, as discussed in Appendix C. They obey the recursion formula [39] Eq. (C3), which we repeat here for the sake of convenience,

$$
\begin{aligned}
x_{j+2}= & \frac{E^{2}+a^{2}+b^{2}-\mu^{2}}{a b} x_{j}-x_{j-2} \\
& +i \mu \frac{b-a}{a b}\left(x_{j-1}-x_{j+1}\right),
\end{aligned}
$$

with the initial values for $x_{N}, y_{N}, y_{N}, \chi_{N}$ given in Table I. To generalize the result in Eq. (G2) to the case including the selfenergies, one should remember that the self-energies act only on the first/last site; the interior of the matrix $\mathcal{M}$ in Eq. (G1)

\footnotetext{
${ }^{2}$ In the strict approach, one has to exclude that $a b=0$ to arrive at the following results. The case of $a b=0$ follows by taking the limit of $a \rightarrow 0$ and/or $b \rightarrow 0$ at the end. The full result is smooth in $a$ and $b$ as one can proof easily. However, in Ref. [64], each inversion of $B$ is countered by a multiplication with $\operatorname{det}(B)$ for cancellation, but both operations enter at different levels in the procedure. Hence, $a b \neq 0$ is only a technical but not a physical restriction.
}

is not affected by them. Importantly, the recursion formula in Eq. (G3) is a consequence of this structure. This justifies an attempt (as it turns out, successful) to solve our problem using Tetranacci polynomials.

Following the technique of Ref. [64], one can define the polynomials $d_{j}^{y}, d_{j}^{x}, d_{j}^{\chi}$, and $d_{j}^{\mathcal{Y}}$ as a superposition of $x_{j}, \mathcal{Y}_{j}$, $y_{j}, \chi_{j}$,

$$
\begin{aligned}
d_{j}^{y} & :=\sigma_{R, p} x_{j-1}+i \sigma_{R, m} y_{j-1}+a y_{j}, \\
d_{j}^{\mathcal{Y}} & :=\sigma_{R, p} \chi_{j-1}+i \sigma_{R, m} \mathcal{Y}_{j-1}+a \mathcal{Y}_{j}, \\
d_{j}^{x} & :=\sigma_{R, p} y_{j-1}-i \sigma_{R, m} x_{j-1}+b x_{j}, \\
d_{j}^{\chi} & :=\sigma_{R, p} \mathcal{Y}_{j-1}-i \sigma_{R, m} \chi_{j-1}+b \chi_{j},
\end{aligned}
$$

including the entries of the right self-energy as coefficients. Physical intuition leads us to believe that, similar to $d_{j}^{y}, d_{j}^{\mathcal{Y}}, d_{j}^{x}$, and $d_{j}^{\chi}$, also Tetranacci polynomials including only the left self-energy exist. Our use of Eqs. (G4)-(G7) is only a matter of the chosen technique.

In the end, one finds

$$
\begin{aligned}
\frac{\operatorname{det}(\mathcal{M})}{(-a b)^{N-1}}= & d_{N}^{y} d_{N}^{\chi}-d_{N}^{x} d_{N}^{\mathcal{Y}} \\
& +\frac{\sigma_{L, m}^{2}-\sigma_{L, p}^{2}}{a b}\left[d_{N-1}^{y} d_{N-1}^{\chi}-d_{N-1}^{x} d_{N-1}^{\mathcal{Y}}\right] \\
& +\frac{\sigma_{L, p}}{b}\left[d_{N}^{y} d_{N-1}^{x}-d_{N}^{x} d_{N-1}^{y}\right] \\
& +\frac{\sigma_{L, p}}{a}\left[d_{N}^{\chi} d_{N-1}^{\mathcal{Y}}-d_{N}^{\mathcal{Y}} d_{N-1}^{\chi}\right] \\
& +i \frac{\sigma_{L, m}}{a}\left[d_{N}^{y} d_{N-1}^{\chi}-d_{N}^{x} d_{N-1}^{\mathcal{Y}}\right] \\
& +i \frac{\sigma_{L, m}}{b}\left[d_{N}^{\mathcal{Y}} d_{N-1}^{x}-d_{N}^{\chi} d_{N-1}^{y}\right]
\end{aligned}
$$

and the entries $G_{1, N+1}^{r}, G_{1, N}^{r}$, and $G_{1,2 N}^{r}$ read

$$
\begin{aligned}
G_{1, N+1}^{r} \frac{2 \operatorname{det}(\mathcal{M})}{(-a b)^{N-2}}= & \frac{b^{2}}{a}\left[d_{N-2}^{\mathcal{Y}} d_{N-1}^{\chi}-d_{N-1}^{\mathcal{Y}} d_{N-2}^{\chi}\right] \\
& +\frac{a^{2}}{b}\left[d_{N-2}^{y} d_{N-1}^{x}-d_{N-1}^{y} d_{N-2}^{x}\right] \\
& +i a\left[d_{N-1}^{\chi} d_{N-2}^{y}-d_{N-1}^{\mathcal{Y}} d_{N-2}^{x}\right] \\
& -i b\left[d_{N-1}^{x} d_{N-2}^{\mathcal{Y}}-d_{N-1}^{y} d_{N-2}^{\chi}\right],
\end{aligned}
$$

$$
\begin{aligned}
G_{1,2 N}^{r} \frac{2 \operatorname{det}(\mathcal{M})}{(-a b)^{N-1}}= & \frac{b}{a}\left[d_{N-2}^{\chi}-i d_{N-2}^{\mathcal{Y}}\right] \\
& -\frac{a}{b}\left[d_{N-2}^{y}+i d_{N-2}^{x}\right] \\
& +\left(E-\Omega_{L,+}-\mu\right) \\
& \times\left[\frac{d_{N-1}^{x}-i d_{N-1}^{y}}{b}-\frac{d_{N-1}^{\mathcal{Y}}+i d_{N-1}^{\chi}}{a}\right],
\end{aligned}
$$

$$
\begin{aligned}
G_{1, N}^{r} \frac{2 \operatorname{det}(\mathcal{M})}{(-a b)^{N-1}}= & \frac{b}{a}\left[d_{N-2}^{\chi}+i d_{N-2}^{\mathcal{Y}}\right] \\
& +\frac{a}{b}\left[d_{N-2}^{y}-i d_{N-2}^{x}\right]
\end{aligned}
$$




$$
\begin{aligned}
& +\left(E-\Omega_{L,+}-\mu\right) \\
& \times\left[\frac{d_{N-1}^{x}+i d_{N-1}^{y}}{b}+\frac{d_{N-1}^{\mathcal{Y}}-i d_{N-1}^{\chi}}{a}\right]
\end{aligned}
$$

The results for $\operatorname{det}(\mathcal{M}), G_{1, N+1}^{r}, G_{1, N}^{r}$, and $G_{1,2 N}^{r}$ hold for all values of $N, t, \Delta, \mu, E$, and the wide band limit is not used yet. Notice that these functions do not diverge at $t= \pm \Delta$, i.e., $a=0$ or $b=0$. The reason is that all denominators contain only $a^{\prime} s$ and $b^{\prime} s$, which are exactly canceled by the prefactors $(-a b)^{N-x}$ for $x=1,2$. This statement is obvious after a look into Eq. (G1), since no entries of the matrix diverge there. Strictly speaking, one has to take the limit $a(b) \rightarrow 0$ and not to evaluate at $a=0(b=0)$, but this is merely a numerical issue.

Taking a closer look to the Andreev contribution in Fig. 7(a), one observes very light and thin lines representing Andreev conduction minima outside the conduction gap, which intertwine with the darker ones, representing the maxima. They are caused by two features of $G_{1, N+1}^{r}$ : first, it contains Tetranacci polynomials $d_{j}$ also for $j=N-1$ and $j=N-2$; second, these polynomials enter here as a product, therefore in a higher order than in $G_{1, N}^{r}$, and at their zeros the Andreev transmission is suppressed more strongly than the direct transmission.

\section{APPENDIX H: CONDUCTANCE FORMULA}

The conductance follows from Eq. (15) by its derivative with respect to the bias in the zero bias limit. In the wide band limit at $T=0 \mathrm{~K}$, we find

$$
\begin{aligned}
G_{D} & =4 \frac{e^{2}}{h} \gamma_{L} \gamma_{R}\left|G_{1, N}^{r}\right|_{E=0}^{2}, \\
G_{A} & =4 \frac{e^{2}}{h} \gamma_{L}^{2}\left|G_{1, N+1}^{r}\right|_{E=0}^{2},
\end{aligned}
$$

and, of course, $G=G_{D}+G_{A}$. The necessary Green's functions are given by Eqs. (G8)-(G11) and we have only to evaluate them at $E=0$. In turn, one should focus first on the Tetranacci polynomials $d_{j}^{y}, d_{j}^{\mathcal{Y}}, d_{j}^{x}, d_{j}^{\chi}$ from Eqs. (G4)-(G7). At $E=0$, they reduce to

$$
\begin{aligned}
\left.d_{j}^{y}\right|_{E=0} & =i \gamma_{R} x_{j-1,0}, \\
\left.d_{j}^{\chi}\right|_{E=0} & =i \gamma_{R} \mathcal{Y}_{j-1,0}, \\
\left.d_{j}^{\mathcal{Y}}\right|_{E=0} & =a \mathcal{Y}_{j, 0}, \\
\left.d_{j}^{x}\right|_{E=0} & =b x_{j, 0} .
\end{aligned}
$$

We use Eq. (C9) to eliminate $\mathcal{Y}_{j, 0}$ and we find in a first step after some algebra that

$$
\begin{aligned}
\left.\operatorname{det}(\mathcal{M})\right|_{E=0} b^{2-2 N}= & b^{2} x_{N, 0}^{2}-x_{N-1,0}^{2}\left(\gamma_{L}^{2}+\gamma_{R}^{2}\right), \\
& +x_{N-2,0}^{2} \frac{\gamma_{L}^{2} \gamma_{R}^{2}}{b^{2}}
\end{aligned}
$$

$$
\begin{aligned}
& -\gamma_{L} \gamma_{R}\left(x_{N-1,0}^{2}-x_{N, 0} x_{N-2,0}\right) \\
& \times \frac{a^{2 N-2}+b^{2 N-2}}{(-a b)^{N-1}} .
\end{aligned}
$$

The key to shorten the last expression and to further simplifications is the function $g_{s}(s= \pm 1)$,

$$
g_{s}:=b^{N-1}\left[i s b x_{N, 0}+i x_{N-1,0}\left(\gamma_{R}-s \gamma_{L}\right)-\frac{i}{b} x_{N-2,0} \gamma_{L} \gamma_{R}\right]
$$

since one gets that

$$
\begin{aligned}
-\left|b^{1-N} g_{s}\right|^{2}= & b^{2} x_{N, 0}^{2}-x_{N-1,0}^{2}\left(\gamma_{R}-s \gamma_{L}\right)^{2} \\
& +x_{N-2,0}^{2} \frac{\gamma_{L}^{2} \gamma_{R}^{2}}{b^{2}} \\
& -2 s \gamma_{L} \gamma_{R} x_{N, 0} x_{N-2,0}
\end{aligned}
$$

which is very close to the expression of $\left.\operatorname{det}(\mathcal{M})\right|_{E=0}$ in Eq. (H3). To obtain the equality in Eq. (H5), one has to use that $s^{2}=1$ and that $x_{j, 0}$ is a real valued function, see Eq. (C5) and Table I. The last identity we need to simplify $\left.\operatorname{det}(\mathcal{M})\right|_{E=0}$ reads

$$
x_{j-1,0}^{2}-x_{j, 0} x_{j-2,0}=\left(-\frac{a}{b}\right)^{j-1},
$$

which follows directly from Eq. (C8) and the fact that $R_{+} R_{-}=-a / b$ with $R_{ \pm}$from Eq. (C7). Adding and subtracting the term $2 s \gamma_{L} \gamma_{R}\left(x_{N-1,0}^{2}-x_{N, 0} x_{N-2,0}\right)$ to $\left.\operatorname{det}(\mathcal{M})\right|_{E=0}$ and using the Eqs. (H5) and (H6) yields

$$
\left.\operatorname{det}(\mathcal{M})\right|_{E=0}=(-1)^{N}\left|g_{s}\right|^{2}-\gamma_{L} \gamma_{R}\left[a^{N-1}+s(-b)^{N-1}\right]^{2},
$$

where a factor $(-1)^{N-1}$ occurs for taking $b^{N-1}$ out of the absolute value in Eq. (H5).

Finally, the simplifications of the entries $G_{1, N+1}^{r}$ and $G_{1, N}^{r}$ at $E=0$ starting from Eqs. (G9)-(G11) read

$$
\begin{array}{r}
\left.G_{1, N}^{r}\right|_{E=0}=(-1)^{N-1} \frac{a^{N-1}+(-b)^{N-1}}{\left.2 \operatorname{det}(\mathcal{M})\right|_{E=0}} g_{-}, \\
\left.G_{1, N+1}^{r}\right|_{E=0}=-i \gamma_{R} \frac{a^{2 N-2}-b^{2 N-2}}{\left.2 \operatorname{det}(\mathcal{M})\right|_{E=0}}, \\
\left.G_{1,2 N}^{r}\right|_{E=0}=(-1)^{N} \frac{a^{N-1}-(-b)^{N-1}}{\left.2 \operatorname{det}(\mathcal{M})\right|_{E=0}} g_{+},
\end{array}
$$

where we give the result of $\left.G_{1,2 N}^{r}\right|_{E=0}$ only for completeness. The use of the Eqs. (H8) and (H9) together with Eqs. (H1) and (H2) yields to the expressions Eqs. (22) and (23), as we show now.

The function $q_{s}$ from Eq. (19) is constructed such that $\left|q_{s}\right|^{2}=\left|g_{s}\right|^{2}$. Further, we have $b=i p, a=-i m$ with $p=$ $t+\Delta$ and $m=t-\Delta$. In a first step, we get for the total conductance $G=G_{D}+G_{A}$,

$$
\begin{aligned}
\frac{\left.|2 \operatorname{det}(\mathcal{M})|_{E=0}\right|^{2}}{4 \gamma_{L} \gamma_{R}} \frac{h}{e^{2}} G & =\left(p^{N-1}+m^{N-1}\right)^{2}\left|g_{-}\right|^{2}+\gamma_{L} \gamma_{R}\left(p^{2 N-2}-m^{2 N-2}\right)^{2} \\
& =\left(p^{N-1}+m^{N-1}\right)^{2}\left[\left|g_{-}\right|^{2}+\gamma_{L} \gamma_{R}\left(m^{N-1}-p^{N-1}\right)^{2}\right]
\end{aligned}
$$


after reorganizing the terms arising from Eqs. (H8) and (H9). The use of Eq. (H7) yields

$$
|\operatorname{det}(\mathcal{M})|_{E=0} \mid=\left[\left|g_{s}\right|^{2}+\gamma_{L} \gamma_{R}\left(m^{N-1}+s p^{N-1}\right)^{2}\right]
$$

and the total conductance becomes

$$
G=\frac{e^{2}}{h} \frac{\gamma_{L} \gamma_{R}\left(p^{N-1}+m^{N-1}\right)^{2}}{\left|g_{+}\right|^{2}+\gamma_{L} \gamma_{R}\left(p^{N-1}+m^{N-1}\right)^{2}} .
$$

Since $\left|g_{s}\right|^{2}=\left|q_{s}\right|^{2}$ holds, we find the conductance according to Eq. (21).

[1] J. Alicea, Phys. Rev. B 81, 125318 (2010).

[2] M. Leijnse and K. Flensberg, Semicond. Sci. Technol. 27, 124003 (2012).

[3] S. R. Elliott and M. Franz, Rev. Mod. Phys. 87, 137 (2015).

[4] R. Aguado, La Rivista del Nuovo Cimento 40, 523 (2017).

[5] S. Das Sarma, M. Freedman, and C. Nayak, npj Quantum Inf. 1, 15001 (2015).

[6] D. Aasen, M. Hell, R. V. Mishmash, A. Higginbotham, J. Danon, M. Leijnse, T. S. Jespersen, J. A. Folk, C. M. Marcus, K. Flensberg, and J. Alicea, Phys. Rev. X 6, 031016 (2016).

[7] T. E. O'Brien, P. Rozek, and A. R. Akhmerov, Phys. Rev. Lett. 120, 220504 (2018).

[8] M. T. Deng, V. S., E. Hansen, J. Danon, M. Leijnse, K. Flensberg, J. Nygård, P. Krogstrup, and C. M. Marcus, Science 354, 1557 (2016).

[9] O. Gül, H. Zhang, J. D. S. Bommer, M. W. A. de Moor, D. Car, S. R. Plissard, E. P. A. M. Bakkers, A. Geresdi, K. Watanabe, T. Taniguchi, and L. P. Kouwenhoven, Nat. Nanotechnol. 13, 192 (2018).

[10] E. Prada, P. San-Jose, M. W. A. de Moor, A. Geresdi, E. J. H. Lee, J. Klinovaja, D. Loss, J. Nygård, R. Aguado, and L. P. Kouwenhoven, Nat. Rev. Phys. 2, 575 (2020).

[11] K. T. Law, P. A. Lee, and T. K. Ng, Phys. Rev. Lett. 103, 237001 (2009).

[12] K. Flensberg, Phys. Rev. B 82, 180516(R) (2010).

[13] C.-X. Liu, J. D. Sau, T. D. Stanescu, and S. Das Sarma, Phys. Rev. B 96, 075161 (2017).

[14] A. Vuik, B. Nijholt, A. R. Akhmerov, and M. Wimmer, SciPost Phys. 7, 61 (2019).

[15] H. Pan and S. Das Sarma, Phys. Rev. Res. 2, 013377 (2020).

[16] O. Dmytruk, D. Loss, and J. Klinovaja, Phys. Rev. B 102, 245431 (2020).

[17] J. Chen, B. D. Woods, P. Yu, M. Hocevar, D. Car, S. R. Plissard, E. P. A. M. Bakkers, T. D. Stanescu, and S. M. Frolov, Phys. Rev. Lett. 123, 107703 (2019).

[18] B. D. Woods, J. Chen, S. M. Frolov, and T. D. Stanescu, Phys. Rev. B 100, 125407 (2019).

[19] M.-T. Deng, S. Vaitiekenas, E. Prada, P. San-Jose, J. Nygård, P. Krogstrup, R. Aguado, and C. M. Marcus, Phys. Rev. B 98, 085125 (2018).

[20] M. Hell, K. Flensberg, and M. Leijnse, Phys. Rev. B 97, 161401(R) (2018).

[21] H. Zhang, D. E. Liu, M. Wimmer, and L. P. Kouwenhoven, Nat. Commun. 10, 5128 (2019).

[22] S. Plugge, A. Rasmussen, R. Egger, and K. Flensberg, New J. Phys. 19, 012001 (2017).

[23] L. Qin, X.-Q. Li, A. Shnirman, and G. Schön, New J. Phys. 21, 043027 (2019).
[24] D. E. Liu and H. U. Baranger, Phys. Rev. B 84, 201308(R) (2011).

[25] J. S. Lim, R. López, and L. Serra, New J. Phys. 14, 083020 (2012).

[26] D. Rainis, L. Trifunovic, J. Klinovaja, and D. Loss, Phys. Rev. B 87, 024515 (2013).

[27] E. Prada, R. Aguado, and P. San-Jose, Phys. Rev. B 96, 085418 (2017)

[28] A. Y. Kitaev, Phys. Usp. 44, 131 (2001).

[29] R. J. Doornenbal, G. Skantzaris, and H. T. C. Stoof, Phys. Rev. B 91, 045419 (2015).

[30] D. Giuliano, S. Paganelli, and L. Lepori, Phys. Rev. B 97, 155113 (2018).

[31] R. V. Mishmash, D. Aasen, A. P. Higginbotham, and J. Alicea, Phys. Rev. B 93, 245404 (2016).

[32] A. Kobiałka, T. Domański, and A. Ptok, Sci. Rep. 9, 12933 (2019).

[33] J. Danon, A. B. Hellenes, E. B. Hansen, L. Casparis, A. P. Higginbotham, and K. Flensberg, Phys. Rev. Lett. 124, 036801 (2020).

[34] J. Ulrich and F. Hassler, Phys. Rev. B 92, 075443 (2015).

[35] X.-Q. Li and L. Xu, Phys. Rev. B 101, 205401 (2020).

[36] S. Hegde, V. Shivamoggi, S. Vishveshwara, and D. Sen, New J. Phys. 17, 053036 (2015).

[37] A. A. Zvyagin, Low Temp. Phys. 41, 625 (2015).

[38] H.-c. Kao, Phys. Rev. B 90, 245435 (2014).

[39] N. Leumer, M. Marganska, B. Muralidharan, and M. Grifoni, J. Phys.: Condens. Matter 32, 445502 (2020).

[40] C.-K. Chiu, J. C. Y. Teo, A. P. Schnyder, and S. Ryu, Rev. Mod. Phys. 88, 035005 (2016).

[41] X. Wen and A. Zee, Nucl. Phys. B 316, 641 (1989).

[42] R. S. K. Mong and V. Shivamoggi, Phys. Rev. B 83, 125109 (2011).

[43] W. Izumida, L. Milz, M. Marganska, and M. Grifoni, Phys. Rev. B 96, 125414 (2017)

[44] K. Kawabata, R. Kobayashi, N. Wu, and H. Katsura, Phys. Rev. B 95, 195140 (2017)

[45] C. W. J. Beenakker, D. I. Pikulin, T. Hyart, H. Schomerus, and J. P. Dahlhaus, Phys. Rev. Lett. 110, 017003 (2013).

[46] S. Das Sarma, J. D. Sau, and T. D. Stanescu, Phys. Rev. B 86, 220506(R) (2012).

[47] B. Pekerten, A. M. Bozkurt, and I. Adagideli, Phys. Rev. B 100, 235455 (2019).

[48] H. Haug and A.-P. Jauho, Quantum Kinetics in Transport and Optics of Semiconductors (Springer, Berlin, 1996).

[49] K. Flensberg and H. Bruus, Introduction to Many-Body Quantum Theory in Condensed Matter Physics (Oxford Graduate Texts, New York, 2002). 
[50] D. A. Ryndyk, Theory of Quantum Transport at Nanoscale (Springer, Cham, Heidelberg, 2016).

[51] J. Rammer and H. Smith, Rev. Mod. Phys. 58, 323 (1986).

[52] Y. Meir and N. S. Wingreen, Phys. Rev. Lett. 68, 2512 (1992).

[53] A. Levy Yeyati, A. Martín-Rodero, and F. J. García-Vidal, Phys. Rev. B 51, 3743 (1995).

[54] M. di Ventra, Electrical Transport in Nanoscale Systems (Cambridge University Press, Cambridge, 2008).

[55] Z. Y. Zeng, B. Li, and F. Claro, Phys. Rev. B 68, 115319 (2003).

[56] P. Sriram, S. S. Kalantre, K. Gharavi, J. Baugh, and B. Muralidharan, Phys. Rev. B 100, 155431 (2019).
[57] R. Mélin, F. S. Bergeret, and A. Levy Yeyati, Phys. Rev. B 79, 104518 (2009).

[58] A. Andreev, JETP 19, 1228 (1964).

[59] G. E. Blonder, M. Tinkham, and T. M. Klapwijk, Phys. Rev. B 25, 4515 (1982).

[60] V. E. jun. Hoggatt and C. T. Long, The Fibonacci Quarterly 12, 113 (1974).

[61] W. Webb and E. Parberry, The Fibonacci Quarterly 7, 457 (1969).

[62] M. Özvatan and O. Pashaev, arXiv:1707.09151.

[63] D. C. Langreth, Linear and Nonlinear Electron Transport in Solids (Springer, New York, London, 1976), Vol. 17.

[64] L. G. Molinari, Linear Algebra Appl. 429, 2221 (2008). 